\title{
Explicit incorporation of deformation twins into crystal plasticity finite element models
}

\author{
Milan Ardeljan ${ }^{\mathrm{a}}$, Rodney J. McCabe ${ }^{\mathrm{b}}$, Irene J. Beyerlein ${ }^{\mathrm{c}}$, Marko Knezevic ${ }^{\mathrm{a}}{ }^{\text {** }}$ \\ ${ }^{a}$ Department of Mechanical Engineering, University of New Hampshire, Durham, NH, 03824, USA \\ ${ }^{b}$ Materials Science and Technology Division, Los Alamos National Laboratory, Los Alamos, NM 87545, USA \\ ${ }^{\mathrm{c}}$ Theoretical Division, Los Alamos National Laboratory, Los Alamos, NM 87545, USA
}

\begin{abstract}
Deformation twinning is a subgrain mechanism that strongly influences the mechanical response and microstructural evolution of metals especially those with low symmetry crystal structure. In this work, we present an approach to modeling the morphological and crystallographic reorientation associated with the formation and thickening of a twin lamella within a crystal plasticity finite element (CPFE) framework. The CPFE model is modified for the first time to include the shear transformation strain associated with deformation twinning. Using this model, we study the stress-strain fields and relative activities of the active deformation modes before and after the formation of a twin and during thickening within the twin, and in the parent grain close to the twin and away from the twin boundaries. These calculations are carried out in cast uranium (U), which has an orthorhombic crystal structure and twins predominantly on the $\{130\}$ $<3 \overline{1} 0>$ systems under ambient conditions. The results show that the resolved shear stresses on a given twin system on the twin-parent grain interface and in the parent are highly inhomogeneous. We use the calculated mechanical fields to determine whether the twin evolution occurs via thickening of the existing twin lamella or formation of a second twin lamella. The analysis suggests that the driving force for thickening the existing twin lamella is low and that formation of multiple twin lamellae is energetically more favorable. The overall modeling framework and insight into why twins in $U$ tend to be thin are described and discussed in this paper.
\end{abstract}

Keywords: Crystal plasticity finite element models; Stress fields; Deformation twinning; Twin formation; Twin thickening

\footnotetext{
* Corresponding author at: University of New Hampshire, Department of Mechanical Engineering, 33 Academic Way, Kingsbury Hall, W119, Durham, New Hampshire 03824, United States. Tel.: 603862 5179; fax: 603862 1865. E-mail address: marko.knezevic@unh.edu (M. Knezevic).
} 


\subsection{Introduction}

Deformation twinning plays a significant role in the deformation response of many metals and their alloys, particularly those with low symmetry crystal structures, such as hexagonal close packed $\mathrm{Mg}, \mathrm{Zr}, \mathrm{Be}, \mathrm{Ti}$ and orthorhombic uranium (U). To accommodate a general deformation state, these polycrystalline metals activate both dislocation slip and twinning [1-9]. The relative resistance and contributions of these two modes can evolve differently with strain and strain direction [4, 10-12]. As a result, these metals exhibit a highly anisotropic mechanical response. Understanding the interplay of slip and twinning at the microscopic level and its influence on the macroscopic response are premier challenges in constitutive modeling of low symmetry materials. Additionally, there are fundamental questions regarding how and where twinning initiates, why a single lamella would thicken in one case while multiple lamellae would form in another, and when twinning saturates.

In the past, many studies have used materials models to link polycrystalline flow stress and hardening to slip and twinning within the individual crystals. Reproducing the effects of deformation twinning is usually more demanding and challenging than for slip. Deformation twins affect the microstructure in two ways: they reorient the lattice in a finite domain within a grain, and they introduce a twin-matrix boundary between the original parent grain and the twin domain [3, 13]. Current models incorporating twins include analytical models [14], polycrystalline mean-field schemes such as Taylor [15-23] and self-consistent models [24, 25], and spatially resolved techniques, such as crystal plasticity finite element (CPFE) [26-34] and Green's function fast Fourier transform (FFT) [35, 36] models. Typically, polycrystalline meanfield models account for the shear produced by twinning by modeling it as a pseudo-slip process $[37,38]$. At the same time, they consider the reorientation effect in one of several ways, either via: (i) the predominant twin reorientation (PTR) method [38], (ii) the volume fraction transfer (VFT) scheme [12], (iii) the total Lagrangian approach [17, 30], or (iv) the composite grain (CG) method [11]. While successful in capturing the macroscopic stress-strain response and bulk texture evolution $[39,40]$, these schemes do not capture the dynamic and spatially heterogeneous nature of twinning. A particularly important limitation is their inability to account for the resolved shear stress at the twin-parent grain interface responsible for thickening of an existing twin.

Spatial full-field techniques can explicitly incorporate the characteristic twin shear and corresponding grain reorientation and, therefore, better capture fields associated with subsequent twin thickening. To understand the local stress distribution as a consequence of twin formation, phenomenological finite element modeling has been utilized to model a twin in $\mathrm{Zr}$ [41, 42]. A cuboidal inclusion representing a parent grain that develops a twin was embedded in an isotropic medium. As such, the effects of morphology and local grain structure on the fields were not taken into account. Moreover plastic anisotropy of the inclusion was accounted for using a macroscopic Hill's yield criterion [43]. The study correlated the stress states that are energetically favorable for twinning with twin volume fraction and morphology. More recently, a full-field, elasto-viscoplastic formulation based on the Green's function FFT method was used to 

variation in the shear stress profile on the twin planes consistent with the lenticular shape of twins. However, modeling of twin thickening and the associated evolution of the mechanical fields have not been modeled using the FFT technique.

Explicit incorporation of deformation twinning in CPFE has not been attempted thus far. With CPFE, a polycrystal is discretized into finite elements and a crystal plasticity constitutive law operates at each FE integration point. Both stress equilibrium and strain compatibility are satisfied providing better predictions of local and overall material behavior and microstructure evolution. In CPFE, one of the main difficulties is related to FE mesh manipulation. Remeshing of every grain containing one twin or multiple twins must be performed at the initial twin formation stage and every step during twin thickening. These mesh alterations computationally intensify the already intensive calculations involved in CPFE codes.

The main objective of this work is to develop a numerical procedure for explicit 3D modeling of the formation and thickening of twin lamellae under imposed plastic deformation conditions into the CPFE framework. The novel procedure explicitly models twin transformation, i.e., the characteristic twin shear and crystallographic reorientation of the twinned domains. Moreover, the effect of both the twin transformation shear and neighboring grain shape in $3 \mathrm{D}$ on the local stress distribution are modeled. In addition to twin formation and thickening, the model captures grain-to-grain interactions and their effect on the mechanical fields. To showcase this capability, we apply the newly developed twin-CPFE model to study twinning in U. Specifically, we treat the case of twin lamellae formation and thickening within a grain in $U$ favorably oriented for twinning, which is surrounded by other grains that are not favorably oriented for twinning. The analysis aims to demonstrate how the technique can provide unique insights into the changes in the stress state and in slip mode activity in the parent and twin grain after the initial twin formation and during twin thickening. As an example, two scenarios for twin expansion are considered: expansion of the first lamella formed or formation of a second lamella and no further expansion of the first lamella. We use resolved shear stress fields and strain energy density arguments to indicate which scenario would be more favorable. For the particular material and microstructure considered here, the latter is favored. The technique developed here is intended as a simulation tool to study the development of deformation twins within a microstructural and mechanical framework.

The paper is structured as follows. In section 2, the modelling framework, including the kinematics and hardening models, is presented. In section 3, the selected material, uranium, for the study is discussed. In section 4 , we present the methodology and computational tools developed to model the formation and thickening of a twin lamella. In section 5, we show and discuss the results of a case study on twin growth in uranium. In section 6 , we finalize the article with a few concluding remarks. 


\subsection{Modeling framework}

A modeling framework is presented here using a notation that is now standard in continuum mechanics. We denote tensors using boldface symbols and not italicized ones, while scalars are italicized and not boldfaced. In this notation, $\mathbf{F}$ represents the deformation gradient tensor, $\mathbf{L}$ represents the velocity gradient tensor, and $\boldsymbol{\sigma}$ represents the Cauchy stress tensor. In this framework, the formulation described below is enforced at each integration point within each element in the finite mesh to relate the stress to the strain work conjugate.

\subsection{Kinematics of slip and twinning within $C P F E$}

The applied velocity gradient tensor, $\mathbf{L}$, is given by

$$
\mathbf{L}=\mathbf{L}^{e}+\mathbf{L}^{p}
$$

where $\mathbf{L}^{e}$ and $\mathbf{L}^{p}$ are the elastic and plastic velocity gradients, respectively. The plastic velocity gradient contains the contributions from slip and twinning via

$$
\mathbf{L}^{p}=\mathbf{L}^{s l}+\mathbf{L}^{t w} .
$$

The expressions for the velocity gradients, due to slip and twinning, are respectively:

$$
\mathbf{L}^{s l}=\sum_{\alpha}^{N^{s l}} \dot{\gamma}^{\alpha} \mathbf{m}_{o}^{\alpha}, \quad \mathbf{L}^{t w}=\sum_{\beta}^{N^{t w}} \dot{f}^{\beta} S^{\beta} \mathbf{m}_{o}^{\beta},
$$

where $\dot{\gamma}^{\alpha}$ is the shearing rate on the slip system $\alpha, \mathbf{m}_{o}^{\alpha}$ and $\mathbf{m}_{o}^{\beta}$ are Schmid tensors of the slip system $\alpha$ and twin system $\beta$, respectively, $S^{\beta}$ is the characteristic twin shear, and $N^{s l}$ and $N^{t w}$ are the total number of available slip and twin systems, respectively. The Schmid tensors are the unit slip (or twin) system tensor, defined as the dyadic product of two orthogonal unit vectors denoting the slip (or twin) shear direction and the slip (or twin) plane normal, respectively. The subscript, $\mathrm{O}^{\prime}$ in the total Lagrangian framework employed in this work indicates that these tensors are defined using the initial crystal orientation and therefore the tensors are known $a$ priori.

The rate of change of the twin volume fraction per twin system, $\dot{f}^{\beta}$, is based on the pseudo slip model [30,38], given by $\dot{f}^{\beta}=\frac{\dot{\gamma}^{\beta}}{s^{\beta}}$, or in a given strain increment by $\Delta f^{\beta}=\frac{\Delta \gamma^{\beta}}{s^{\beta}}$. If in $n$ strain increments, the volume fraction of a particular twin system, $\beta$, reaches unity, i.e., $\sum_{1}^{n} \Delta f^{\beta}=1$, then

$$
S^{\beta}=\sum_{1}^{n} \Delta \gamma^{\beta}
$$

The pseudo slip model accounts for twinning shear strains in the same manner as it does for slip. It only considers the shear accommodated by twinning, but does not account for the reorientation or the formation of a twin domain.

For finite deformations, the total deformation gradient tensor $(\mathbf{F})$ can be decomposed into elastic and plastic components as:

$$
\mathbf{F}=\mathbf{F}^{e} \mathbf{F}^{p}
$$


where $\mathbf{F}^{e}$ contains deformation gradients due to both elastic stretching and lattice rotation, while $\mathbf{F}^{p}$ denotes the deformation gradient because of plastic deformation alone. The evolution of $\mathbf{F}^{p}$ can be expressed in a rate form using the following flow rule relationship:

$$
\dot{\mathbf{F}}^{p}=\mathbf{L}^{p} \mathbf{F}^{p} \text {. }
$$

After integration from $t$ to $\tau=t+\Delta t$, Eq. (6) becomes:

$$
\mathbf{F}^{p}(\tau)=\exp \left(\mathbf{L}^{p} \Delta t\right) \mathbf{F}^{p}(t) .
$$

We can further approximate the exponential as:

$$
\mathbf{F}^{p}(\tau)=\left\{\mathbf{I}+\Delta t \mathbf{L}^{p}\right\} \mathbf{F}^{p}(t)=\left\{\mathbf{I}+\Delta t\left(\mathbf{L}^{s l}+\mathbf{L}^{t w}\right)\right\} \mathbf{F}^{p}(t),
$$

where $\mathbf{I}$ is the identity matrix. The constitutive equation for stress in the crystal is expressed as:

$$
\mathbf{T}^{e}=\mathbf{C E}^{e}, \mathbf{T}^{e}=\mathbf{F}^{e^{-1}}\left\{\left(\operatorname{det} \mathbf{F}^{e}\right) \boldsymbol{\sigma}\right\} \mathbf{F}^{e^{-T}}, \mathbf{E}^{e}=\frac{1}{2}\left\{\mathbf{F}^{e^{T}} \mathbf{F}^{e}-\mathbf{I}\right\},
$$

where $\mathbf{C}$ is the fourth-order elasticity tensor, $\mathbf{T}^{e}$ is the second Piola-Kirchhoff stress, which is elastic work conjugate to the Lagrangian finite strain $\mathbf{E}^{e}$, and $\boldsymbol{\sigma}$ is the Cauchy stress in the crystal. To calculate stress, we need to evaluate $\mathbf{F}^{e}=\mathbf{F} \mathbf{F}^{p^{-1}}$. Therefore we can rewrite Eq. (8) as:

$$
\mathbf{F}^{p^{-1}}(\tau)=\mathbf{F}^{p^{-1}}(t)\left\{\mathbf{I}-\Delta t\left(\mathbf{L}^{s l}+\mathbf{L}^{t w}\right)\right\} .
$$

Next, we divide $\mathbf{L}^{t w}$ into two parts, $\mathbf{L}^{t w, p t s}$ and $\mathbf{L}^{t w, o t s}$, i.e.,

$$
\mathbf{L}^{t w}=\mathbf{L}^{t w, p t s}+\mathbf{L}^{t w, o t s} \text {. }
$$

In Eq. (11), $\mathbf{L}^{t w, p t s}$ is the velocity gradient of the most active variant, which we will call the predominant twin system (pts) and $\mathbf{L}^{t w, o t s}$ is the velocity gradient from all other twin systems (ots) contributing to plasticity.

\subsection{Kinetics of the slip and twinning mechanism}

In order to estimate the shear strain rate, $\dot{\gamma}^{\alpha}$, for each slip system $\alpha$, and the shear strain rate, $\dot{\gamma}^{\beta}$, for each twinning system $\beta$, we can relate the resolved shear stress $\left(\tau^{\alpha}=\mathbf{T}^{e} \cdot \mathbf{m}_{0}^{\alpha}\right.$ for slip and $\tau^{\beta}=\mathbf{T}^{e} \cdot \mathbf{m}_{0}^{\beta}$ for twinning) on the system to the characteristic resistance $\tau_{c}^{\alpha}$ for slip systems and $\tau_{c}^{\beta}$ for twin systems according to the following power-law relationships [30, 44, 45]:

$$
\dot{\gamma}^{\alpha}=\dot{\gamma}_{0}\left(\frac{\left|\tau^{\alpha}\right|}{\tau_{c}^{\alpha}}\right)^{\frac{1}{m}} \operatorname{sign}\left(\tau^{\alpha}\right), \dot{\gamma}^{\beta}=\left\{\begin{array}{cc}
\dot{\gamma}_{0}\left(\frac{\left|\tau^{\beta}\right|}{\tau_{c}^{\beta}}\right)^{\frac{1}{m}} \operatorname{sign}\left(\tau^{\beta}\right) & \text { if } \tau^{\beta}>0 \\
0 & \text { if } \tau^{\beta}<0
\end{array}\right.
$$

where $\dot{\gamma}_{0}$ is a reference slip rate (arbitrarily taken here as $0.001 \mathrm{~s}^{-1}$ ) and $m$ represents the strain rate sensitivity factor (taken here to be 0.02 for both slip and twinning systems). 


\subsection{Hardening laws for slip and twinning}

In this work, we use a dislocation density hardening law formulation to compute the evolution of the slip and twin resistances as a function of strain, temperature, and strain rate [40, 46]. It is assumed that all slip and twin systems within the same deformation mode share the same values for critical resolved shear stress (CRSS). This hardening law has been successfully used to model deformation of several metals within mean-field self-consistent codes, differing in crystal structure, such as Haynes 25 [47], AA6022 [48], Nb [49, 50], Ta [51, 52], Mg [53-55], Zr $[46,56,57], \mathrm{Be}[10,58]$, and $\mathrm{U}[4,59,60]$. Here, we integrate the same hardening law for $\mathrm{U}$ in CPFE and enable the existing CPFE to model the orthorhombic structure. Below is a brief review of this model.

The critical resolved shear stress for activation of slip considers contributions of several different terms: a friction stress $\tau_{0, f}^{\alpha}$, a forest dislocation interaction stress $\tau_{f o r}^{\alpha}$ and a dislocation substructure interaction stress $\tau_{\text {sub }}^{\alpha}$ :

$$
\tau_{c}^{\alpha}=\tau_{0, f}^{\alpha}+\tau_{\text {for }}^{\alpha}+\tau_{\text {sub }}^{\alpha} \text {. }
$$

The model for the critical resolved shear stress for twin activation is distinct from that for slip. It accounts for a temperature-independent friction term $\tau_{0}^{\beta}$ and a latent hardening term coupling the active slip and the twin systems. Taken together, the critical resolved shear stress for twinning can be expressed as:

$$
\tau_{c}^{\beta}=\tau_{0}^{\beta}+\mu^{\beta} \sum_{\alpha} C^{\alpha \beta} b^{\beta} b^{\alpha} \rho_{\text {for }}^{\alpha} .
$$

Here $\mu^{\beta}, b^{\beta}$ and $C^{\alpha \beta}$ represent the elastic shear modulus, Burgers vector on the given twin system, and the latent hardening matrix used for coupling of slip and twin systems, respectively. The behavior of $\tau_{f o r}^{\alpha}$ and $\tau_{s u b}^{\alpha}$ is governed by the evolution of the dislocation densities in the form of forest $\rho_{\text {for }}^{\alpha}$ and substructure $\rho_{\text {sub }}^{\alpha}$ dislocations. These relationships, for each dislocation type, can be expressed in the form of a Taylor law:

$$
\begin{gathered}
\tau_{\text {for }}^{\alpha}=\chi b^{\alpha} \mu^{\alpha} \sqrt{\rho_{\text {for }}^{\alpha}} \text {, and } \\
\tau_{\text {sub }}^{\alpha}=k_{\text {sub }} \mu^{\alpha} b^{\alpha} \sqrt{\rho_{\text {sub }}} \log \left(\frac{1}{b^{\alpha} \sqrt{\rho_{\text {sub }}}}\right)
\end{gathered}
$$

where $\chi=0.9$ is a dislocation interaction parameter, $\mu^{\alpha}$ is the shear modulus and $k_{\text {sub }}=0.086$ is a mathematical parameter that insures that Eq. (15) recovers the Taylor law at low dislocation densities [61]. Equation (16) is related to substructure stress required for gliding dislocations to bow out and punch through arrays of locked dislocation segments that are closely spaced at later stages of hardening [61]. The stored forest density $\rho_{\text {for }}^{\alpha}$ evolves via a competition between the rate of storage and the rate of dynamic recovery:

$$
\begin{aligned}
& \frac{\partial \rho_{\text {for }}^{\alpha}}{\partial \gamma^{\alpha}}=\frac{\partial \rho_{\text {gen,for }}^{\alpha}}{\partial \gamma^{\alpha}}-\frac{\partial \rho_{\text {rem,for }}^{\alpha}}{\partial \gamma^{\alpha}}=k_{1}^{\alpha} \sqrt{\rho_{\text {for }}^{\alpha}}-k_{2}^{\alpha}(\dot{\varepsilon}, \mathrm{T}) \rho_{\text {for }}^{\alpha}, \\
& \Delta \rho_{f o r}^{\alpha}=\frac{\partial \rho_{\text {for }}^{\alpha}}{\partial \gamma^{\alpha}}\left|\Delta \gamma^{\alpha}\right|
\end{aligned}
$$



dislocations by the forest obstacles and $k_{2}^{\alpha}$ is the coefficient for the rate of dynamic recovery, which is given by the following expression: [46]

$$
\frac{k_{2}^{\alpha}(\dot{\varepsilon}, \mathrm{T})}{k_{1}^{\alpha}}=\frac{\chi b^{\alpha}}{g^{\alpha}}\left(1-\frac{k T}{D^{\alpha} b^{3}} \ln \left(\frac{\dot{\varepsilon}}{\dot{\varepsilon}_{0}}\right)\right) .
$$

In Eq. (19), $k, \dot{\varepsilon}_{0}, g^{\alpha}$ and $D^{\alpha}$ are respectively Boltzmann's constant, a reference strain rate, an effective activation enthalpy and a drag stress. The drag stress for dislocation apply to dislocation speeds within the thermally activated regime where phonon effects can be reasonably neglected [62]. It has been proposed and suggested via discrete dislocation dynamics simulations that the processes accountable for dynamic recovery are similar to those that lead to the formation of dislocation substructures, patterns of dislocations, such as cell walls and dislocation sheets. Dislocation interactions can result in the formation of jogs, dipoles, and lock that selforganize under deformation into relatively low energy-structures [63]. In our model, the increment in substructure development is proportional to the rate of dynamic recovery of all active dislocations and can be expressed as:

$$
\Delta \rho_{s u b}=\sum_{\alpha} q b^{\alpha} \frac{\partial \rho_{r e m, f o r}^{\alpha}}{\partial \gamma^{\alpha}}\left|\Delta \gamma^{\alpha}\right|
$$

where $q$ is a coefficient defining the fraction of an $\alpha$-type dislocations that do not annihilate but become substructure dislocation. The hardening parameters have been calibrated in the earlier study [59] and will be used here.

\subsection{Deformation mechanisms of uranium}

Under the ambient conditions modeled here, $U$ has a base-centered orthorhombic crystal structure, where the unit cell dimensions are $a=0.2852, b=0.5865$ and $c=0.4945 \mathrm{~nm}$. Because of the low crystallographic symmetry, $U$ deforms by both slip and twinning mechanisms $[8,9,64,65]$. The most dominant slip modes observed in $U$ are (010)[100] and (001)[100], with only one independent slip system in each. The two other slip modes found active in $U$ are $\frac{1}{2}\{110\}\langle 1 \overline{1} 0\rangle$ and $\frac{1}{2}\{021\}\langle 1 \overline{1} 2\rangle$, with two and four slip systems, respectively. The prominent deformation twin in $U$ is $\{130\}\langle 3 \overline{1} 0\rangle$, and the value of its characteristic twin shear is 0.299 . The $\{172\}\langle 3 \overline{1} 2\rangle$ twin mode, and its reciprocal twin $\{112\}\langle 3 \overline{7} 2\rangle$ mode both with characteristic shears of 0.227 , have also been observed. The characteristic twinning shear values are based on crystallography and are taken from $[3,8,9]$. The, $\{130\}\langle 3 \overline{1} 0\rangle$ and $\{172\}\langle 3 \overline{1} 2\rangle$ twin modes reorient the crystal lattice by $69.3^{\circ}$ about [001] and $92.6^{\circ}$ about $<\overline{10} 70>$, respectively. Specific geometries of the U slip and twin systems are shown in Fig. 1. Figure 2 shows orientation maps based on the electron backscattered diffraction (EBSD) of a deformed microstructure of cast $U$ at a strain of 0.05. In slight contrast to the typical EBSD image of deformed $\mathrm{Mg}$ alloys [5], $\mathrm{Zr}$ [57], or Ti [39], we observe a large number of relatively thin twin lamellae per grain in cast U. The origin of this unusual twin morphology has yet to be understood. 


\subsection{Explicit incorporation of twinning in CPFE}

The flow chart in Fig. 3 illustrates the overall procedure for twin formation and thickening within CPFE. This procedure is explained next.

\subsection{Finite element model of the grain structure}

A synthetic microstructure is generated first in the publicly available software DREAM.3D $[66,67]$. We find that DREAM.3D generates grains that are more realistic in terms of grain morphology and grain size distribution than those typically generated using the Voronoi tessellation scheme. It overcomes several limitations of Voronoi tessellation. Generally, the Voronoi tessellation begins with random seeds, which produces a near-equiaxed set of domains (or grains). It is possible to place seeds far apart in one dimension to get elongated grains, but it is difficult to control the aspect ratios. Also, with Voronoi tessellations it is difficult to obtain size or shape distributions that have "heavy tails". The distributions of these parameters tend to be fairly normally distributed by the nature of the process. Furthermore, because the boundary between the two domains/grains is placed halfway between the seed points, it is challenging to obtain grain neighbors of disparate sizes, i.e., large grains next to small grains. Lastly, Voronoi tessellations yield perfectly planar boundaries, which may be significantly different from those of real grain boundaries.

DREAM.3D generates 3D synthetic voxel-based microstructure and surface meshes for each individual grain based on a specified grain size and grain shape distributions. Furthermore, we define voxel density/resolution in the model in order to achieve the desired number of surface finite elements. A surface mesh is the bridge between a voxel-based model and a volumetric (in our case tetrahedral) mesh, and is the foundation for successful 3D mesh generation. Starting from the surface mesh, we perform 3D solid meshing of individual grains ensuring mesh conformance between grain boundaries using Patran [68]. We refer to this as ,conformal' grain boundaries, an aspect that is critical to modeling grain-to-grain interactions and is not found in other spatially resolved, full-field techniques such as the Green's function FFT method [35]. The reader can find a detailed step-by-step explanation of this meshing procedure in [28]. Figure 3a shows the final voxel-based and meshed microstructures used in the present study. In order to unveil the interior of the model and to show the grain morphologies, we cut the model in half and expose two cut planes (see the two right-most images in Fig. 4).

The FE model of the 3D microstructure contains 27 grains and approximately 480000 finite elements (each grain consists of approximately 18000 finite elements). As shown, these large U grains are packed in a cube with an edge length of $1.0 \mathrm{~mm}$. In order to discretize the whole volume, we use linear tetrahedral elements each with one integration point (type C3D4 in ABAQUS). In our previous study [28], we performed a detailed analysis regarding the selection of the elements type and size. From there, we observed that the use of higher-order elements (e.g. C3D10M) does not significantly affect the distribution of stress-strain fields or the prediction of texture and grain boundaries. Because these microstructures consist of grains with highly 

computationally less intensive, overcomes the benefits of meshing with a smaller number of higher order elements, which are computationally more intensive.

We arbitrarily assign crystal orientations that would not develop substantial twin activity to every grain except the red grain in the center of the FE model. The crystal orientation of this grain is selected such that it favors the twinning system (130)[3 $\overline{1} 0$ ] under simple compression along the normal direction, ND (the axis $\mathrm{Z}$ in Fig. 4). The particular orientation defined by Bunge-Euler angles is $\left(0^{\circ}, 90^{\circ}, 10^{\circ}\right)$. Crystal orientations of other grains do not develop twins during the early stage of simple compression deformation. The same crystal orientation is assigned to each finite element within a corresponding grain, which results in the initial intragranular misorientations of zero for each grain.

The simple compression is performed by imposing displacement in the $\mathrm{Z}$ direction (or the normal direction, ND) with the lateral sides traction-free and, hence, free to expand (Fig. 3a). The compression consists of several deformation steps, necessary to initially form and then thicken the twin lamella. It was interrupted at three different strain levels corresponding to (1) $1 \%$ of a twin variant volume fraction, (2) $3 \%$ volume fraction thickening of the twin lamella, and (3) $5 \%$ volume fraction thickening of the twin lamella. When the twin volume fraction of the predominant twin system (pts) reaches a threshold value of $1 \%$ of the central grain's volume an initial twin lamella is formed. Next, we describe the model for twin formation and thickening.

\subsection{Procedure for incorporating twin lamellae in an FE mesh}

Explicitly including a twin lamella into the finite element framework requires development of an automated procedure. The procedure is detailed in the flowchart in Fig. 3 and as shown, consists of several geometric manipulations and mesh generations. We have developed a script in Matlab [69], which writes Patran and Python script files containing specific commands for Patran and Abaqus, respectively. The Patran scripts are executed within Patran to perform either surface or solid meshing and the Python script files are executed within Abaqus to either extract the surface mesh from the solid mesh model or to perform the Abaqus mesh cleanup procedure [70]. The final result of this procedure is a twin lamella of a desired volume inserted in a selected parent grain that maintains the overall conformity with the parent and neighboring grains.

After a deformation step, the procedure starts by obtaining the surface mesh of the deformed grains. This step applies regardless of whether a new twin lamella is first formed or a preexisting twin lamella thickens for a prescribed volume increment. Extraction of the surface mesh is achieved using a Python script written by the Matlab script containing the appropriate commands for Abaqus. The output of this Python script is surface meshes of all deformed grains. At this point the procedure continues with either forming or thickening a twin lamella. This step is shown in the Fig. 3b and $\mathrm{c}$ of the flow chart. The Matlab script finds the intersection points between the two cutting planes (that form a twin) and the 3D surface mesh of the parent grain and prepares a Patran script. The Patran script uses coordinates of these points for each intersected plane and connects them into a single closed loop or "chain" consisting of line 
segments. The script contains the commands to generate and export surface meshes for each intersection plane. It is important to mention that these newly generated triangular elements are of the same size as the ones that build the surface of the intersected grain. The control is then returned to the Matlab script. To preserve the mesh conformity of the grain boundaries of neighboring grains touching the twin, the surface meshes of these neighboring grains are also adjusted (Fig. 3d). At this point, we assemble the model that is made of individual grain surface meshes. This model has only grain boundaries that are conformal. Next, the Matlab script writes a Python script for surface mesh cleanup in Abaqus to collapse any elements with bad aspect ratio. The mesh repair procedure collapses elements with the edge aspect ratio greater than 4 . Note that the mesh repair procedure is not performed on individual grain surface meshes because that would ruin the overall mesh conformity between grains when assembling them over the surface mesh of the entire model. The Patran script is then generated and called upon to perform 3D meshing for each individual grain and twin. This step starts from the surface meshes to ensure the overall conformity of the mesh. Finally, the Matlab script writes an Abaqus input file containing the new mesh, state variables, and boundary conditions for the subsequent deformation step.

In carrying out the above procedure, careful attempts are made to minimize changes made to the model microstructure. After meshing, the following state variables: $\mathbf{F}^{p}$, dislocation densities and crystal orientations (preserving any developed intra-grain misorientation), are mapped onto the new mesh. For this mapping, we used a scheme to find the closest elements from the "old" mesh (before forming or growing the twin) to the "newly" created elements (after meshing) and assigning the state variables from the old mesh to the new mesh based on the mutual proximity of the element centroids. In that sense, the overall deformation state of the model and crystal orientations are transferred and preserved from the "old" to the "new" mesh.

The frequency of remeshing procedures is important in order to capture the changes that occur in the stress-strain fields in the material due to twin formation and thickening, which are relevant to twin evolution. In the case of uranium, twin thickening of $2 \%$ volume fraction was determined to be sufficient to reveal the fields. Due to the very fine mesh within every grain in the model, remapping of the state variables from the "old" mesh to the "new" mesh is performed with success, which will be later shown in the evolution of the stress fields in the parent and twin grain at different values of twin volume fraction.

This entire procedure is repeated on every occasion when we want to form new twin lamellae or to grow pre-existing twin lamellae.

\subsubsection{Twin lamella formation}

After the volume fraction of the $\beta=t w$,pts in a grain reaches a pre-specified threshold value, we insert a twin lamella. In the present study, the threshold value of $1 \%$ is chosen. The volume fraction of the $\beta=t w$,pts is calculated as the integral of the $\beta=t w, p t s$ volume fraction over every element set representing a grain (after convergence is achieved in each strain increment). When the threshold is reached, the deformation is interrupted in order to insert the lamella. Note that 

strain increment and checked whether the threshold is reached.

The geometry of the created lamella is uniquely determined by the twin plane normal of the $\beta$ $=t w$,pts twin variant and the thickness of the lamella, which is calculated to produce a specified total twin volume fraction, which in this work is $1 \%$. The twin planes span the entire grain.

\subsubsection{Location of twin lamella formation}

To create the twin lamella, an important question concerns where to place it within the parent grain. Twins are thought to originate from grain boundaries at points of highest stress concentration [71]. To determine the location of the highest stress concentration on the surface of the parent grain, we use the von Mises stress normalized by the average value calculated over the entire volume of the model. Figure 5a shows the contours over the grain, Fig. 5b the actual location where the initial twin lamella has been placed, and Fig. 5 c-f the orientation relationship between the stress concentrations and the location of the twin lamella. In this example, the highest stress concentration lies at the grain boundary where the parent grain meets three other neighboring grains, (i.e., a quadruple point). Starting from this point, we form a twin lamella by inserting two parallel planes across the parent grain with a specified separation to match the twin volume fraction. The planes have a normal vector corresponding to the twin plane of the pts and the twin shear is imposed within this plane in the direction of the Burgers vector of the twin.

\subsubsection{Characteristic twin shear accommodation by the twin lamella}

Thus far, we have discussed one important aspect of the model, which is the ability to introduce a discrete twin lamella within a grain with a twin volume fraction based on the total twinning activity accumulated within the grain. For this we described the needed geometric and FE mesh manipulations. To fully model the twin, we must also consider the other aspect of twinning, which is its transformation into a reoriented volume with a characteristic twin shear. To this end, we describe how we account for both the reorientation and shearing action imposed by the created twin lamella. Specifically, the kinematics and kinetics related to the twin shear strain and the behavior of the parent grain immediately before and after are discussed.

In the freshly formed twin as well as during subsequent twin thickening, $\mathbf{F}^{p}(\tau)$ in the twin is enforced to be:

$$
\mathbf{F}^{p^{-1}}(\tau)=\left\{\mathbf{I}-S^{t w, p t s} \mathbf{m}_{0}^{t w, p t s}\right\}
$$

Next, the deformation within the parent grain needs to be updated. A twin that is forming and growing within a grain contributes to accommodating the strain applied to the grain. Consequently, the plastic deformation in the parent grain will change according to:

$$
\mathbf{F}^{p^{-1}}(\tau)=\mathbf{F}^{p^{-1}}(t)\left\{\mathbf{I}+f^{t w, p t s} S^{t w, p t s} \mathbf{m}_{0}^{t w, p t s}\right\}
$$


where $f^{t w, p t s}$ is the accumulated volume fraction of the twin. The superscript denotes the variant $\beta=t w$,pts that has been selected. Eqns. 21 and 22 together represent the transfer of strain after a twin has formed. Eq. 21 ensures that the strain accommodated by this variant is transferred to the twin and produces the appropriate thickness, while Eq. 22 ensures that it is removed from the parent grain. For numerical stability, the update of $\mathbf{F}^{p}$ in the parent grain and twin at each FE integration point is carried out using a specified number of much smaller strain (or time) increments than that used to simulate the increment in the applied simple compression deformation. The updated values of $\mathbf{F}^{p}$ for all elements within the parent grain and twin are enforced simultaneously during this ,,intermediate period' and while the entire grain structure model is held under the applied simple compression strain.

\subsection{Twin thickening}

As we apply more strain to the model, a twin is permitted to expand in prescribed increments from the initial $1 \%$ twin volume fraction. For demonstration, we consider thickening the initial twin to volume fractions of $3 \%$ and $5 \%$. For this, the FE mesh is altered in a similar manner as twin formation. Maintaining the same twin normal and location of the initially formed twin, we cut the surface mesh of the parent grain by two parallel planes with a specified separation in order to expand the twin volume (Fig. 3). In this case, the surface mesh of the parent grain is now reduced for the volume of the previously formed twin lamella. The distance between the cutting planes is changed in order to reflect the twin growth. Also, the characteristic shear strain of the twin is enforced using Eqs. (19 and 20). Expansion is performed on both sides of the twin until the prescribed twin volume is achieved. For the sake of simplicity, the same twin normal used for twin formation is maintained during twin growth. It is important to emphasize, however, that in our numerical framework, the twin boundary could take on a different normal during growth. Figures 6 and 7 show the parent grain and the grain cluster model after the twin lamella is first created and after it grows to $3 \%$ twin volume fraction.

\subsection{Application: A case study of twinning in uranium}

Having described the CPFE-twinning model, we now, in this section, apply it to simulate the initial formation and thickening of twin lamellae during mechanical loading. We consider simple compression of the $U$ grain structure shown in Fig. 4. The crystal orientation of the central (red) grain was chosen to be favorably oriented for twinning, whereas those of the surrounding grains were chosen to be unfavorably oriented. This arrangement allows us to demonstrate twin modeling in the central (parent) grain only, and to analyze the stress fields in the twin, parent grain, and non-twinning neighbors. We will also discuss the evolution of slip activity within the parent grain before and after twinning and in the twin lamellae as it evolves.

The incremental deformation steps were 0.05 compressive true strain up to the point where $1 \%$ twin volume fraction accumulates. At this stage, the $1 \%$ volume fraction twin lamella was created (Section 4.2). As described earlier (Section 4.2.2), the twin location is determined by points of high von Mises stress concentration in the boundary. Afterwards, the incremental 

twin volume fraction accumulates (according to the pseudo-slip model for twinning). At this point, we expand the original twin from $1 \%$ to $3 \%$ volume fraction (Section 4.3).

Figure 5 compares the normalized Von Mises stress distributions during deformation before twinning and as a function of twin volume fraction after twinning. The stress distributions are heterogeneous, reflecting the effect of interactions between neighboring grains differing in crystallography. In particular, high stress concentrations are seen to develop in the neighboring grains in the vicinity where the twin lamella intersects the grain boundary. These are localized areas where the neighboring grain is accommodating the twin shear produced by the twin lamella.

To identify the areas of high probability of twin formation and thickening, in Fig. 8 we show the contour plots of resolved shear stresses on the most dominant twinning plane and in the twin direction (denoted as RSSTW) as a function of twin volume fraction. These resolved shear stresses are normalized by the current value of twin resistance. As expected, large RSSTW values develop in the center grain, confirming that it is oriented well for twinning, while the relatively low RSSTW values in the neighboring grains show that they are not. After forming the $1 \%$ twin, the driving force for twin propagation decreases in the regions where the twin-matrix interface intersects the grain boundary. This reduction can be seen by the drop in RSSTW below 1.0. Therefore, additional straining must be applied such that the RSSTW reaches 1.0 in these areas. After a true strain increment of 0.07 is applied, twinning activity accumulates corresponding to twin growth from $1 \%$ to $3 \%$. Figs. $9 \mathrm{a}$ and $\mathrm{b}$ show the resolved shear stress on the interface just before expansion to $3 \%$ volume fraction. After twin thickening to $3 \%$, the RSSTW decreases suggesting that continued twin expansion might be hindered. At the same time, the RSSTW value inside the twin is zero or negative (Fig. 9c). However, away from the twin-matrix interface and within the matrix region, the driving force is high $(\mathrm{RSSTW}=1)$ suggesting that nucleation of another lamella may be favored over continued thickening of the $3 \%$ twin. In other words, if the stress were maintained or increased, accommodating more strain via an increase in twin volume fraction is more likely to occur by nucleating a second twin lamella than by expanding the original twin lamella.

To investigate the likelihood of thickening the first twin lamella versus formation of a second twin lamella, we create one model for thickening the existing twin lamella and another model for forming a separate but parallel twin lamella of the same twin variant while not thickening the existing twin lamella. A strain increment of 0.06 strain was sufficient to accumulate the twin volume fractions from $3 \%$ to $5 \%$. Figure 10 shows the two models, which both produce a total of $5 \%$ twin volume fraction. One model consists of growing the $3 \%$ twin to a $5 \%$ twin and the other model contains the original $3 \%$ twin and a second separate $2 \%$ twin. The second twin was placed in a region where the stress concentrations in the grain boundaries were high. Figure 11 shows the normalized von Mises contours. As before, stress concentrations at the tips of the twin lamellae within neighboring grains are evident. The RSSTW contours are shown in Fig. 12. 
In order to assess which scenario is more likely to occur, we first consider changes in plastic work $\Delta \mathrm{W}_{s}$ associated with the transformation from a $3 \%$ twin lamella to the new twin state, $s$, which is generically given by:

$$
\left(\Delta \mathrm{W}_{s}=\frac{1}{V} \sum_{1}^{n} \int_{V} \sigma_{i j} \varepsilon_{i j}^{P}\right)
$$

where $V$ is the domain of integration. The two work terms of interest are $\Delta \mathrm{W}_{1}$, associated with growth to a single twin lamella at $5 \%$ and the other $\Delta \mathrm{W}_{2}$, associated with the state consisting of two lamellae, the original that remains at $3 \%$ and a new one at $2 \%$. We can further consider the difference in these two work terms, i.e.,

$$
\left(\Delta \mathrm{W}=\Delta \mathrm{W}_{1}-\Delta \mathrm{W}_{2}\right) \text {. }
$$

In calculating $\Delta \mathrm{W}_{1}$ and $\Delta \mathrm{W}_{2}$, the increments in plastic strain $\varepsilon_{i j}^{P}$, go from 1 to $n$ and we consider the states $s$ after the same amount of applied deformation. We repeat calculation of these work terms for three domains $V$ for the integration: the whole grain cluster, only the parent and twin lamellae combined, and the parent grain and twin lamellae separately. The results are listed in Table I. As shown, the value of $\Delta \mathrm{W}$ for all three domain types $V$ suggest that forming a secondary twin lamella with $2 \%$ volume fraction is more energetically favorable than increasing the volume fraction of the original one by $2 \%$.

The foregoing application considers just one of many questions relevant to twin growth. There are many possible scenarios of twin growth, such as nucleation of many fine twins, subsequent transmission into the neighboring grains, merging of neighboring twins, etc. The likelihood of each event would depend on the local stress fields imposed by the twins, parent, and neighboring grains while under deformation. Thus, testing for these possibilities calls for a technique such as the one developed here that can simultaneously treat the formation and expansion of discrete twin lamellae in a polycrystal calculation and provide spatially resolved mechanical fields. For demonstration, we considered the possibility of two twin configurations yielding the same twin volume fraction of 5\%. Comparing the energy densities indicates that that generation of secondary twin lamella is more likely to occur. This prediction is consistent with experimental observations that a large number of fine twin lamellae often form within $U$ grains (See Fig. 2). However, we should note that this is not expected to apply in general. Other effects such as parent grain morphology, other grain-neighborhoods, and the number, location and placement of other twin lamellae in 3D space are important factors impacting twin growth. A systematic study of these factors is possible with this technique, but beyond the scope of the present work.

Last, we apply the model to study the accompanying changes in slip activity due to the presence of the discrete twin lamellae. In U, there are four slip modes that can be activated within the parent grain and twin lamellae. Formation of a twin within a grain can affect the choice of slip modes with in the twin. To elucidate any possible changes, we present in Fig. 13 
the contours of the most active slip modes. The plots reflect the accumulated value of relative activities for all four slip modes "incrementally". By this, we mean that at the end of each deformation step for each finite element we identify the slip mode with the highest slip activity within that increment. From the contour plots a change in the predominant slip mode from the $\frac{1}{2}\{021\}<1 \overline{1} 2>$ mode to the (010)[100] mode within the parent grain can be seen when the twin first forms. As the twin lamella expands or a second twin lamella forms, the (010)[100] mode remains predominant in the parent. Within the twin lamellae, the prevalent modes are $\frac{1}{2}\{110\}<1 \overline{1} 0>$ and (001)[100]. Interestingly, at the twin-matrix interface there is a shift between the most active slip modes, suggesting a possible loss of the twin-matrix orientation relationship with deformation, which is yet another reason for hampering the existing twin lamella from thickening. By comparing Figs. 13d and e, we can see that the slip activity within the parent and twin lamellae does not change whether one or two lamellae form.

In closing we mention that while the study presented here has focused on fundamental understanding of changes in stress-strain fields due to twin formation and thickening, extending the framework to calculations of many grains that twin is left for future work. The developed procedure is fully automated; however, to facilitate calculations involving thousands of grains, significant increases in computation speed are necessary. To this end, we plan to explore the use of non-iterative numerical methods that are based on fast Fourier transforms [20, 33, 72-77] and utilization of specialized computer hardware $[18,78]$ that involves graphic hardware.

\subsection{Conclusions}

In this paper, we present a novel approach for modelling deformation twinning by explicit incorporation of twin lamellae into a 3D crystal plasticity finite element framework. The framework is applied to uranium, a material with an orthorhombic crystal structure. Effects of the microstructural characteristics such as grain structure and grain boundaries are explicitly modeled. A 3D representation of uranium with a large grain microstructure is generated using DREAM.3D and a finite element grain model is constructed using a custom tool set. Formation and thickening of a discrete twinned region within the parent grain, which is associated with crystallographic reorientation and characteristic twin shear, is handled with explicit formation and growth of a twin lamella in the finite element framework. To this end, a rigorous procedure is developed for geometry and mesh manipulation associated with twin formation and thickening. Distributions of normalized equivalent and resolved shear stresses are investigated within the parent and twin grain as a function of twin volume in order to elucidate the material response to twin formation and thickening. The calculated mechanical fields are used to determine whether the twin evolution would occur via thickening of the existing twin lamella or formation of a second twin lamella. Furthermore, we analyze the distributions of the most active slip modes. The results suggested that at $5 \%$ twin volume fraction, the twin will most likely form a secondary lamella as opposed to continued growth of the first (original) lamella. To our 
knowledge this is a unique numerical procedure that is able to relate spatially resolved fields of stress, strains with microstructural changes during twin formation and thickening.

\section{Acknowledgements}

M.A. wishes to acknowledge support by the G.T. Seaborg Institute for Transactinium Science through the Seaborg Summer Research Fellowship Program. M.K. acknowledges subcontract, NO. 277871, granted by Los Alamos National Laboratory to the University of New Hampshire. I.J.B. and R.J.M gratefully acknowledge support by a Laboratory Directed Research and Development grants 20140348ER and 20140630ER, respectively.

\section{References}

[1] L. Balogh, S. Niezgoda, A. Kanjarla, D. Brown, B. Clausen, W. Liu, C. Tomé, Spatially resolved in situ strain measurements from an interior twinned grain in bulk polycrystalline AZ31 alloy, Acta Materialia, 61 (2013) 3612-3620.

[2] M. Yoo, J. Lee, Deformation twinning in hcp metals and alloys, Philosophical Magazine A, 63 (1991) 987-1000.

[3] J.W. Christian, S. Mahajan, Deformation twinning, Progress in Materials Science, 39 (1995) $1-157$.

[4] M. Knezevic, L. Capolungo, C.N. Tomé, R.A. Lebensohn, D.J. Alexander, B. Mihaila, R.J. McCabe, Anisotropic stress-strain response and microstructure evolution of textured $\alpha$-uranium, Acta Materialia, 60 (2012) 702-715.

[5] M. Knezevic, A. Levinson, R. Harris, R.K. Mishra, R.D. Doherty, S.R. Kalidindi, Deformation twinning in AZ31: Influence on strain hardening and texture evolution, Acta Materialia, 58 (2010) 6230-6242.

[6] H. Abdolvand, M. Majkut, J. Oddershede, S. Schmidt, U. Lienert, B.J. Diak, P.J. Withers, M.R. Daymond, On the deformation twinning of MgAZ31B: a three-dimensional synchrotron Xray diffraction experiment and crystal plasticity finite element model, International Journal of Plasticity, (2015).

[7] G.Y. Chin, W.F. Hosford, D.R. Mendorf, Accommodation of Constrained Deformation in f.c.c. Metals by Slip and Twinning, Proceedings of the Royal Society of London Series AMathematical and Physical Sciences, 309 (1969) 433-456.

[8] R.W. Cahn, Twinning and slip in $\alpha$-uranium, Acta Crystallographica, 4 (1951) 470.

[9] R.W. Cahn, Plastic deformation of alpha-uranium; twinning and slip, Acta Metallurgica, 1 (1953) 49-70.

[10] M. Knezevic, I.J. Beyerlein, D.W. Brown, T.A. Sisneros, C.N. Tomé, A polycrystal plasticity model for predicting mechanical response and texture evolution during strain-path changes: Application to beryllium, International Journal of Plasticity, 49 (2013) 185-198.

[11] G. Proust, C.N. Tomé, G.C. Kaschner, Modeling texture, twinning and hardening evolution during deformation of hexagonal materials, Acta Materialia, 55 (2007) 2137-2148.

[12] C.N. Tomé, R.A. Lebensohn, U.F. Kocks, A model for texture development dominated by deformation twinning: Application to zirconium alloys, Acta Metallurgica et Materialia, 39 (1991) 2667-2680.

[13] I.J. Beyerlein, X. Zhang, A. Misra, Growth Twins and Deformation Twins in Metals, Annual Review of Materials Research, 44 (2014) 329-363. 

metals, International Journal of Plasticity, 22 (2006) 1171-1194.

[15] G.I. Taylor, Plastic strain in metals, Journal of the Institute of Metals, 62 (1938) 307-324. [16] B.S. Fromm, B.L. Adams, S. Ahmadi, M. Knezevic, Grain size and orientation distributions: Application to yielding of $\alpha$-titanium, Acta Materialia, 57 (2009) 2339-2348.

[17] X. Wu, S.R. Kalidindi, C. Necker, A.A. Salem, Prediction of crystallographic texture evolution and anisotropic stress-strain curves during large plastic strains in high purity $\alpha$ titanium using a Taylor-type crystal plasticity model, Acta Materialia, 55 (2007) 423-432.

[18] M. Knezevic, D.J. Savage, A high-performance computational framework for fast crystal plasticity simulations, Computational Materials Science, 83 (2014) 101-106.

[19] M. Knezevic, S.R. Kalidindi, R.K. Mishra, Delineation of first-order closures for plastic properties requiring explicit consideration of strain hardening and crystallographic texture evolution, International Journal of Plasticity, 24 (2008) 327-342.

[20] M. Knezevic, H.F. Al-Harbi, S.R. Kalidindi, Crystal plasticity simulations using discrete Fourier transforms, Acta Materialia, 57 (2009) 1777-1784.

[21] M. Knezevic, S.R. Kalidindi, D. Fullwood, Computationally efficient database and spectral interpolation for fully plastic Taylor-type crystal plasticity calculations of face-centered cubic polycrystals, International Journal of Plasticity, 24 (2008) 1264-1276.

[22] T. Fast, M. Knezevic, S.R. Kalidindi, Application of microstructure sensitive design to structural components produced from hexagonal polycrystalline metals, Computational Materials Science, 43 (2008) 374-383.

[23] X. Wu, G. Proust, M. Knezevic, S.R. Kalidindi, Elastic-plastic property closures for hexagonal close-packed polycrystalline metals using first-order bounding theories, Acta Materialia, 55 (2007) 2729-2737.

[24] R.A. Lebensohn, C.N. Tomé, A self-consistent anisotropic approach for the simulation of plastic deformation and texture development of polycrystals: Application to zirconium alloys, Acta Metallurgica et Materialia, 41 (1993) 2611-2624.

[25] P.A. Turner, C.N. Tomé, A study of residual stresses in Zircaloy-2 with rod texture, Acta Metallurgica et Materialia, 42 (1994) 4143-4153.

[26] S.R. Kalidindi, C.A. Bronkhorst, L. Anand, Crystallographic texture evolution in bulk deformation processing of FCC metals, Journal of the Mechanics and Physics of Solids, 40 (1992) 537-569.

[27] F. Roters, P. Eisenlohr, L. Hantcherli, D.D. Tjahjanto, T.R. Bieler, D. Raabe, Overview of constitutive laws, kinematics, homogenization and multiscale methods in crystal plasticity finiteelement modeling: Theory, experiments, applications, Acta Materialia, 58 (2010) 1152-1211.

[28] M. Knezevic, B. Drach, M. Ardeljan, I.J. Beyerlein, Three dimensional predictions of grain scale plasticity and grain boundaries using crystal plasticity finite element models, Computer Methods in Applied Mechanics and Engineering, 277 (2014) 239-259.

[29] B.L. Hansen, J.S. Carpenter, S.D. Sintay, C.A. Bronkhorst, R.J. McCabe, J.R. Mayeur, H.M. Mourad, I.J. Beyerlein, N.A. Mara, S.R. Chen, G.T. Gray Iii, Modeling the texture evolution of $\mathrm{Cu} / \mathrm{Nb}$ layered composites during rolling, International Journal of Plasticity, 49 (2013) 71-84.

[30] S.R. Kalidindi, Incorporation of deformation twinning in crystal plasticity models, Journal of the Mechanics and Physics of Solids, 46 (1998) 267-271.

[31] M. Knezevic, M. Jahedi, Y.P. Korkolis, I.J. Beyerlein, Material-based design of the extrusion of bimetallic tubes, Computational Materials Science, 95 (2014) 63-73. 

plasticity framework in implicit finite elements, Mechanics of Materials, 84 (2015) 114-126.

[33] M. Zecevic, R.J. McCabe, M. Knezevic, Spectral database solutions to elasto-viscoplasticity within finite elements: Application to a cobalt-based FCC superalloy, International Journal of Plasticity, 70 (2015) 151-165.

[34] M. Jahedi, M. Ardeljan, I.J. Beyerlein, M.H. Paydar, M. Knezevic, Enhancement of orientation gradients during simple shear deformation by application of simple compression, Journal of Applied Physics, 117 (2015) 214309.

[35] R.A. Lebensohn, A.K. Kanjarla, P. Eisenlohr, An elasto-viscoplastic formulation based on fast Fourier transforms for the prediction of micromechanical fields in polycrystalline materials, International Journal of Plasticity, 32-33 (2012) 59-69.

[36] M. Arul Kumar, A.K. Kanjarla, S.R. Niezgoda, R.A. Lebensohn, C.N. Tomé, Numerical study of the stress state of a deformation twin in magnesium, Acta Materialia, 84 (2015) 349358.

[37] G.Y. Chin, W.L. Mamme, Generalization and Equivalence of the Minimum Work (Taylor) and Maximum Work (Bishop-Hill) Principles for Crystal Plasticity, Transactions of the Metallurgical Society of AIME 245 (1969) 1211-1214.

[38] P. Van Houtte, Simulation of the rolling and shear texture of brass by the Taylor theory adapted for mechanical twinning, Acta Metallurgica et Materialia, 26 (1978) 591-604.

[39] M. Knezevic, R.A. Lebensohn, O. Cazacu, B. Revil-Baudard, G. Proust, S.C. Vogel, M.E. Nixon, Modeling bending of $\alpha$-titanium with embedded polycrystal plasticity in implicit finite elements, Materials Science and Engineering: A, 564 (2013) 116-126.

[40] I.J. Beyerlein, R.J. McCabe, C.N. Tome, Stochastic processes of $\{1012\}$ deformation twinning in hexagonal close-packed polycrystalline zirconium and magnesium, International Journal for Multiscale Computational Engineering, 9 (2011) 459-480.

[41] R.Y. Zhang, M.R. Daymond, R.A. Holt, A finite element model of deformation twinning in zirconium, Materials Science and Engineering: A, 473 (2008) 139-146.

[42] R.Y. Zhang, M.R. Daymond, R.A. Holt, Parametric study of stress state development during twinning using 3D finite element modeling, Materials Science and Engineering: A, 528 (2011) 2725-2735.

[43] R. Hill, A theory of the yielding and plastic flow of anisotropic metals, Proceedings of the Royal Society of London. Series A, Mathematical and Physical Sciences, 193 (1948) 281-297. [44] R.J. Asaro, A. Needleman, Texture development and strain hardening in rate dependent polycrystals, Acta Metallurgica et Materialia, 33 (1985) 923-953.

[45] J.W. Hutchinson, Bounds and self-consistent estimates for creep of polycrystalline materials, Proceedings of the Royal Society of London. Series A, Mathematical and Physical Sciences, (1976) 101-126.

[46] I.J. Beyerlein, C.N. Tomé, A dislocation-based constitutive law for pure $\mathrm{Zr}$ including temperature effects, International Journal of Plasticity, 24 (2008) 867-895.

[47] M. Knezevic, J.S. Carpenter, M.L. Lovato, R.J. McCabe, Deformation behavior of the cobalt-based superalloy Haynes 25: Experimental characterization and crystal plasticity modeling, Acta Materialia, 63 (2014) 162-168.

[48] M. Zecevic, M. Knezevic, A dislocation density based elasto-plastic self-consistent model for the prediction of cyclic deformation: Application to Al6022-T4, International Journal of Plasticity, 10.1016/j.ijplas.2015.05.018, (2015). 

evolution in two-phase $\mathrm{Zr} / \mathrm{Nb}$ lamellar composites during accumulative roll bonding, International Journal of Plasticity, 57 (2014) 16-28.

[50] M. Ardeljan, I.J. Beyerlein, M. Knezevic, A dislocation density based crystal plasticity finite element model: Application to a two-phase polycrystalline HCP/BCC composites, Journal of the Mechanics and Physics of Solids, 66 (2014) 16-31.

[51] M. Knezevic, I.J. Beyerlein, M.L. Lovato, C.N. Tomé, A.W. Richards, R.J. McCabe, A strain-rate and temperature dependent constitutive model for BCC metals incorporating nonSchmid effects: Application to tantalum-tungsten alloys, International Journal of Plasticity, 62 (2014) 93-104.

[52] A. Bhattacharyya, M. Knezevic, M. Abouaf, Characterization of Crystallographic Texture and Intra-Grain Morphology in Cross-Rolled Tantalum, Metallurgical and Materials Transactions A, 46 (2015) 1085-1096.

[53] I.J. Beyerlein, R.J. McCabe, C.N. Tomé, Effect of microstructure on the nucleation of deformation twins in polycrystalline high-purity magnesium: A multi-scale modeling study, Journal of the Mechanics and Physics of Solids, 59 (2011) 988-1003.

[54] M. Lentz, M. Klaus, I.J. Beyerlein, M. Zecevic, W. Reimers, M. Knezevic, In situ X-ray diffraction and crystal plasticity modeling of the deformation behavior of extruded $\mathrm{Mg}-\mathrm{Li}-(\mathrm{Al})$ alloys: An uncommon tension-compression asymmetry, Acta Materialia, 86 (2015) 254-268.

[55] M. Lentz, M. Klaus, M. Wagner, C. Fahrenson, I.J. Beyerlein, M. Zecevic, W. Reimers, M. Knezevic, Effect of age hardening on the deformation behavior of an Mg-Y-Nd alloy: In-situ Xray diffraction and crystal plasticity modeling, Materials Science and Engineering: A, 628 (2015) 396-409.

[56] M. Knezevic, I.J. Beyerlein, T. Nizolek, N.A. Mara, T.M. Pollock, Anomalous Basal Slip Activity in Zirconium under High-strain Deformation, Materials Research Letters, 1 (2013) 133140.

[57] M. Knezevic, M. Zecevic, I.J. Beyerlein, J.F. Bingert, R.J. McCabe, Strain rate and temperature effects on the selection of primary and secondary slip and twinning systems in HCP $\mathrm{Zr}$, Acta Materialia, 88 (2015) 55-73.

[58] M. Zecevic, M. Knezevic, I.J. Beyerlein, C.N. Tomé, An elasto-plastic self-consistent model with hardening based on dislocation density, twinning and de-twinning: Application to strain path changes in HCP metals, Materials Science and Engineering: A, 638 (2015) 262-274.

[59] M. Knezevic, R.J. McCabe, C.N. Tomé, R.A. Lebensohn, S.R. Chen, C.M. Cady, G.T. Gray Iii, B. Mihaila, Modeling mechanical response and texture evolution of $\alpha$-uranium as a function of strain rate and temperature using polycrystal plasticity, International Journal of Plasticity, 43 (2013) 70-84.

[60] M. Knezevic, R.J. McCabe, R.A. Lebensohn, C.N. Tomé, C. Liu, M.L. Lovato, B. Mihaila, Integration of self-consistent polycrystal plasticity with dislocation density based hardening laws within an implicit finite element framework: Application to low-symmetry metals, Journal of the Mechanics and Physics of Solids, 61 (2013) 2034-2046.

[61] L. Capolungo, I.J. Beyerlein, C.N. Tomé, Slip-assisted twin growth in hexagonal closepacked metals, Scripta Materialia, 60 (2009) 32-35.

[62] Z. Wang, I. Beyerlein, Stress orientation and relativistic effects on the separation of moving screw dislocations, Physical Review B, 77 (2008) 184112.

[63] D. Kuhlmann-Wilsdorf, Theory of plastic deformation:-properties of low energy dislocation structures, Materials Science and Engineering: A, 113 (1989) 1-41. 
[64] J.S. Daniel, B. Lesage, P. Lacombe, The influence of temperature on slip and twinning in uranium, Acta Metallurgica, 19 (1971) 163-173.

[65] R.J. McCabe, L. Capolungo, P.E. Marshall, C.M. Cady, C.N. Tomé, Deformation of wrought uranium: Experiments and modeling, Acta Materialia, 58 (2010) 5447-5459.

[66] M. Groeber, S. Ghosh, M.D. Uchic, D.M. Dimiduk, A framework for automated analysis and simulation of 3D polycrystalline microstructures. Part 2: Synthetic structure generation, Acta Materialia, 56 (2008) 1274-1287.

[67] DREAM.3D Version 4.2, BlueQuartz Software, Springboro, OH, USA, (2013).

[68] Patran Version 2013, MSC Software Corporation, Newport Beach, CA, USA, (2013).

[69] MATLAB Version R2012a, The MathWorks Inc., Natick, MA, USA, (2013).

[70] ABAQUS Version 6.11-2, Dassault Systèmes, Providence, RI, USA, (2013).

[71] R.L. Bell, R. Cahn, The dynamics of twinning and the interrelation of slip and twinning in zinc crystals, Proceedings of the Royal Society of London. Series A. Mathematical and Physical Sciences, 239 (1957) 494-521.

[72] J.B. Shaffer, M. Knezevic, S.R. Kalidindi, Building texture evolution networks for deformation processing of polycrystalline fcc metals using spectral approaches: Applications to process design for targeted performance, International Journal of Plasticity, 26 (2010) 11831194.

[73] S.R. Kalidindi, M. Knezevic, S. Niezgoda, J. Shaffer, Representation of the orientation distribution function and computation of first-order elastic properties closures using discrete Fourier transforms, Acta Materialia, 57 (2009) 3916-3923.

[74] H.F. Al-Harbi, M. Knezevic, S.R. Kalidindi, Spectral approaches for the fast computation of yield surfaces and first-order plastic property closures for polycrystalline materials with cubictriclinic textures, CMC: Computers, Materials, \& Continua, 15 (2010) 153-172.

[75] M. Knezevic, N.W. Landry, Procedures for reducing large datasets of crystal orientations using generalized spherical harmonics, Mechanics of Materials, 88 (2015) 73-86.

[76] S.R. Kalidindi, H.K. Duvvuru, M. Knezevic, Spectral calibration of crystal plasticity models, Acta Materialia, 54 (2006) 1795-1804.

[77] M. Knezevic, S.R. Kalidindi, Fast computation of first-order elastic-plastic closures for polycrystalline cubic-orthorhombic microstructures, Computational Materials Science, 39 (2007) 643-648.

[78] B. Mihaila, M. Knezevic, A. Cardenas, Three orders of magnitude improved efficiency with high - performance spectral crystal plasticity on GPU platforms, International Journal for Numerical Methods in Engineering, 97 (2014) 785-798. 
Captions:

Figure 1 Slip and twin systems in uranium. The arrow shows the Burgers vector direction in the plane.

Figure 2 EBSD-based scan of tension direction orientation map of the microstructure for the cast uranium studied here. The colors in the orientation map represent the orientation of the ND sample direction with respect to the grain orientations.

Figure 3 Modeling framework for explicit incorporation of twinning in CPFE.

Figure 4 A finite element model of an explicit grain structure consisting of 27 grains and 480000 C3D4 elements. The two sections on the right show the internal grain structure. The red grain in the center has a crystallographic orientation selected to favor $\{130\}<3 \overline{1} 0>$ twinning.

Figure 5 Distributions of the von Mises stress ( $\overline{\mathrm{VM} i s e s}$ ) normalized by the applied stress over the parent grain (a) without a twin lamella, (b) after the formation of $1 \%$ twin lamella, (c-d) Y-Z sections (c) before and (d) after 1\% twin formation, and (e-f) X-Z sections (e) before and (f) after $1 \%$ twin formation.

Figure 6 (a) FE mesh of the grain favorably oriented for twinning. (b) Parent and twin grain after creating the twin lamella of $1 \%$ volume fraction. (c) Parent grain and twin after twin growth from $1 \%$ to $3 \%$ volume fraction. Note the small change in shape of the grain under deformation. The original FE mesh of the parent grain has not changed substantially after the twin lamella is introduced except in close proximity to the twin lamella.

Figure 7 Twin formation and thickening with respect to the surrounding neighboring grains in the 3D model: (a) initial grain structure, (b) grain structure with $1 \%$ formed twin and (c) grain structure with $3 \%$ twin.

Figure 8 Distributions of normalized resolved shear stresses $(\overline{\text { RSSTW }})$ along the most dominant twin plane in the twin direction for different twin volume percentage (a) no twin, (b) $1 \%$ twin, and (c) $3 \%$ twin. Note that neighboring grains do not exhibit the propensity for twinning.

Figure 9 Distributions of normalized resolved shear stress ( $\overline{\text { RSSTW }})$ along (a) the left interfacial planes between matrix and the twin lamella, (b) the right interfacial planes between matrix and the twin lamella, (c) a plane in the middle of the twin, and (d) slightly away in the matrix region from the right interface where the second twin lamella has formed. Different stages of deformation are shown as indicated in the figure. 
Figure 10 Grain structures with a parent grain containing (a) one 5\% twin lamella and (b) two lamellae one of $2 \%$ and another of $3 \%$ volume fraction.

Figure 11 Distributions of normalized value of equivalent stresses ( $\left.\overline{V_{M} i s e s}\right)$ for $5 \%$ twin volume fraction for one lamella twin and two twin lamellae.

Figure 12 Distributions of normalized resolved shear stresses $(\overline{\mathrm{RSSTW}})$ along the most dominant twin plane in the twin direction for different $5 \%$ twin volume fraction (a) one lamella and (b) two lamellae.

Figure 13 Distributions of the most active slip modes at (a) no twin, (b) $1 \%$ twin, (c) $3 \%$ twin, (d) $5 \%$ one lamella twin and (e) 5\% two lamellae twin. Slip mode IDs: 1. (010)[100], 2. $\frac{1}{2}\{110\}<1 \overline{1} 0>, 3$. (001) [100], 4. $\frac{1}{2}\{021\}<1 \overline{1} 2>$.

Table I Comparison of plastic work calculated over the volume indicated in the table with one lamella vs. two twin lamellae. 


\begin{tabular}{|c|c|c|c|c|c|}
\hline \hline 1010)[100] & $(001)[100]$ & $\frac{1}{2}\{110\}\langle 1 \overline{1} 0\rangle$ & $\frac{1}{2}\{021\}\langle 1 \overline{1} 2\rangle$ & $\begin{array}{c}\{130\}\langle 3 \overline{1} 0\rangle \\
\text { 2 Slip System }\end{array}$ & $\begin{array}{c}\{172\}\langle 3 \overline{1} 2\rangle \\
\text { 1 Slip System }\end{array}$ \\
\hline
\end{tabular}

Figure 1 Slip and twin systems in uranium. The arrow shows the Burgers vector direction in the plane.

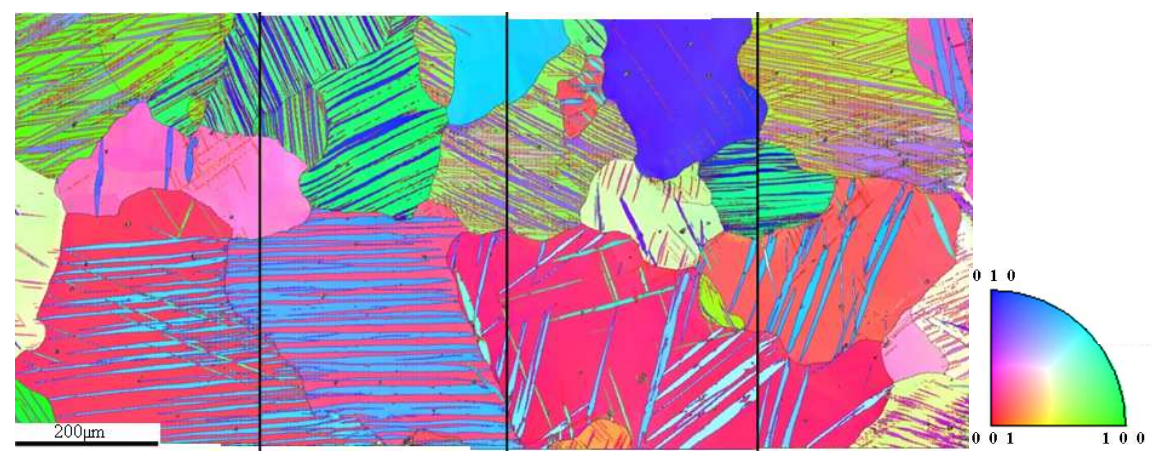

Figure 2 EBSD-based scan of tension direction orientation map of the microstructure for the cast uranium studied here. The colors in the orientation map represent the orientation of the ND sample direction with respect to the grain orientations. 


\section{Create voxel-based polycrystalline \\ (a) microstructures}

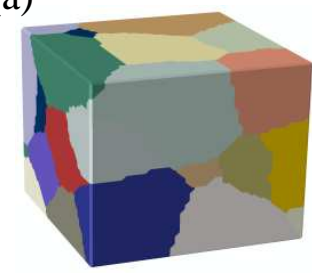

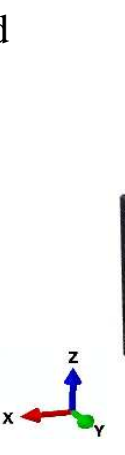

\section{Define finite} element mesh of grain structure

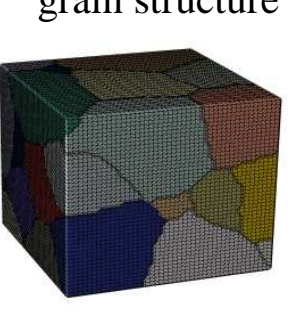

Perform deformation (simple compression)

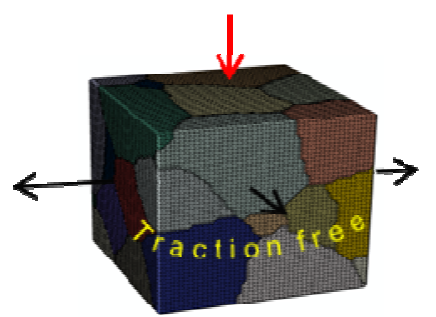

Extract surface mesh of individual grains

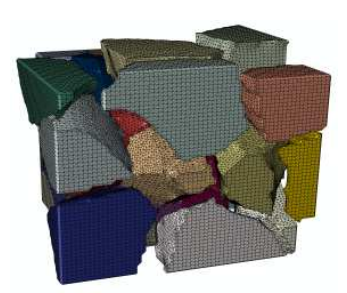

\section{Twin formation}

Select grains developing $f^{t w, p t s}$
Define twin planes corresponding to $\beta=t w$, pts
Intersect grain surface mesh with twin planes
Generate surface mesh over parent grain and twin (b)

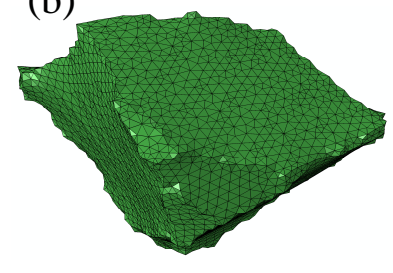

$\underbrace{z}_{1}$

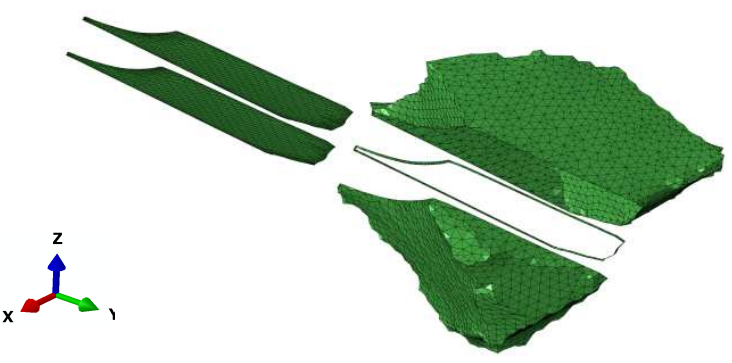

\section{Twin thickening}

Select grains with growing $f^{t w, p t s}$

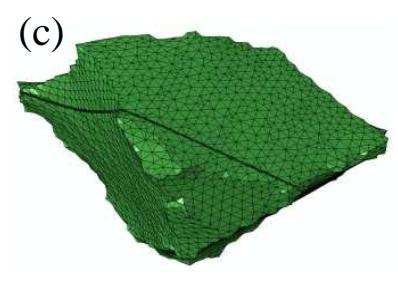

Define twin planes corresponding to $\beta=t w$, pts

Intersect grain surface

Generate surface mesh mesh with twin planes

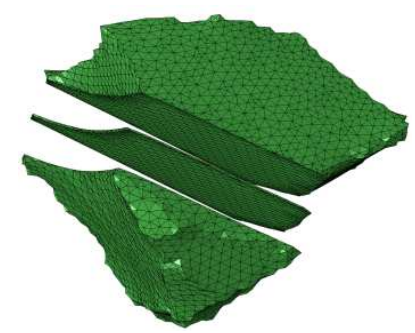

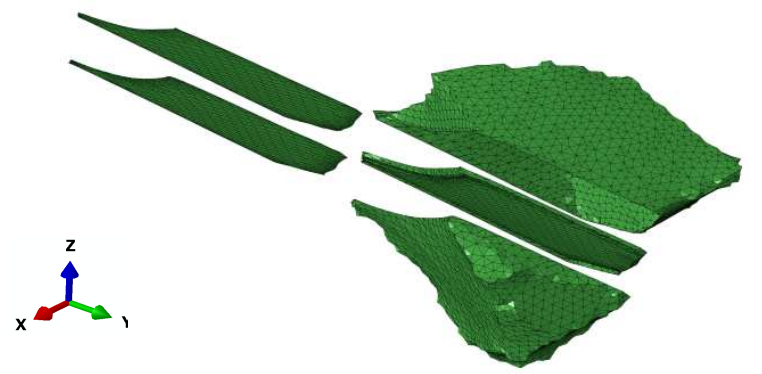

Assemble mesh and perform mesh repair

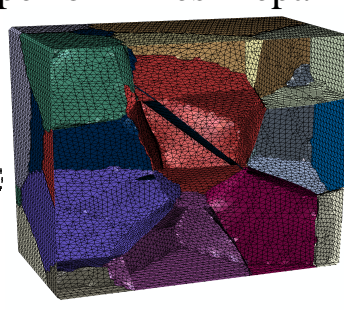

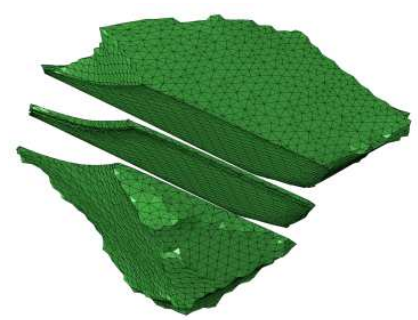

Mesh and map state variables

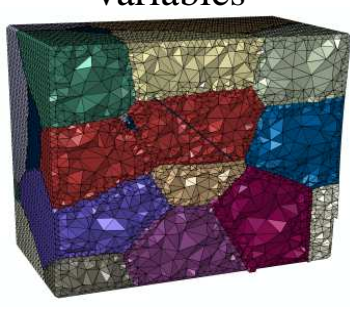

Figure 3 Modeling framework for explicit incorporation of twinning in CPFE. 

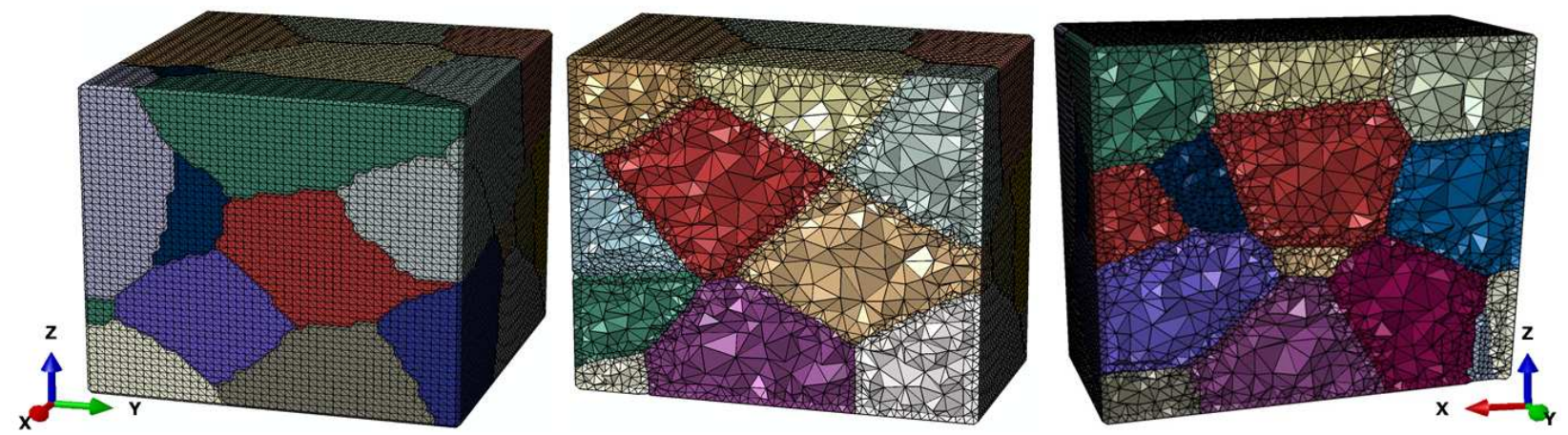

Figure 4 A finite element model of an explicit grain structure consisting of 27 grains and 480000 C3D4 elements. The two sections on the right show the internal grain structure. The red grain in the center has a crystallographic orientation selected to favor $\{130\}<3 \overline{1} 0\rangle$ twinning.

(a)

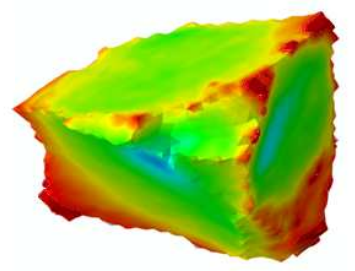

(c)

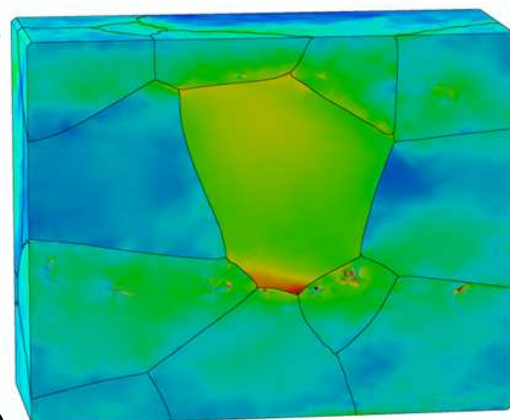

(e)

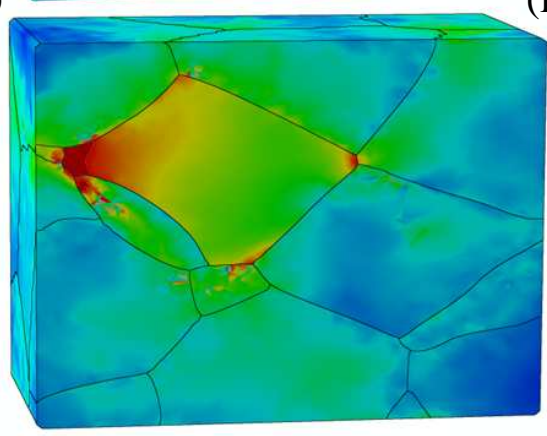

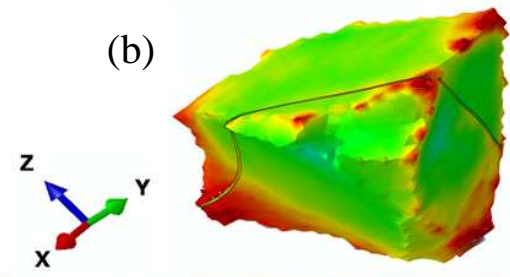

(d)

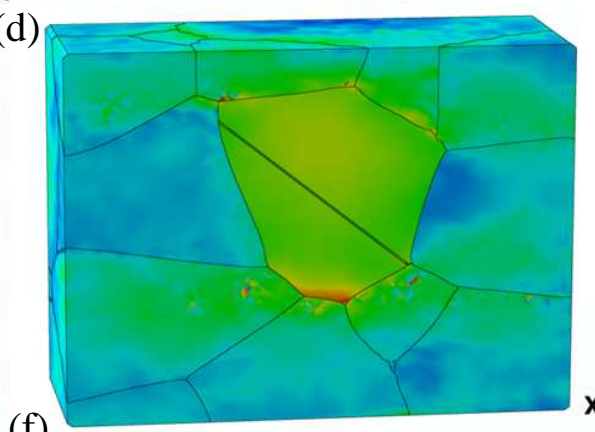

(f)

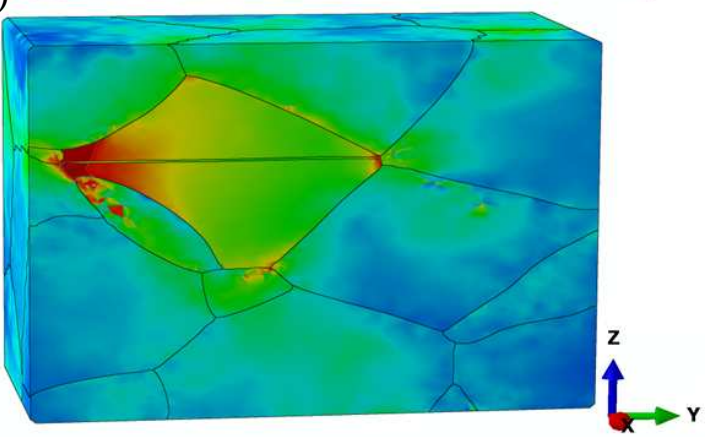

Figure 5 Distributions of the von Mises stress ( $\overline{\mathrm{VM} i s e s}$ ) normalized by the applied stress over the parent grain (a) without a twin lamella, (b) after the formation of $1 \%$ twin lamella, (c-d) Y-Z sections (c) before and (d) after $1 \%$ twin formation, and (e-f) X-Z sections (e) before and (f) after $1 \%$ twin formation. 
(a)

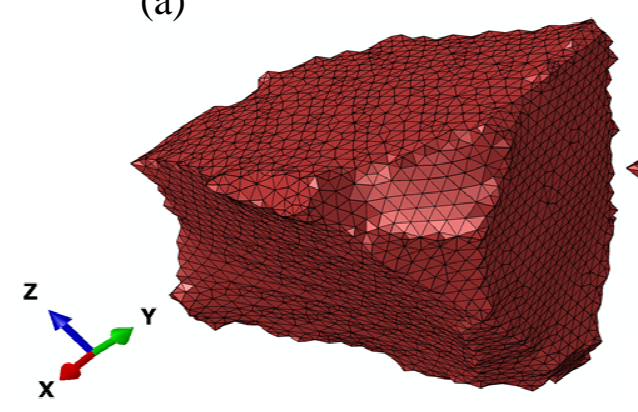

(b)

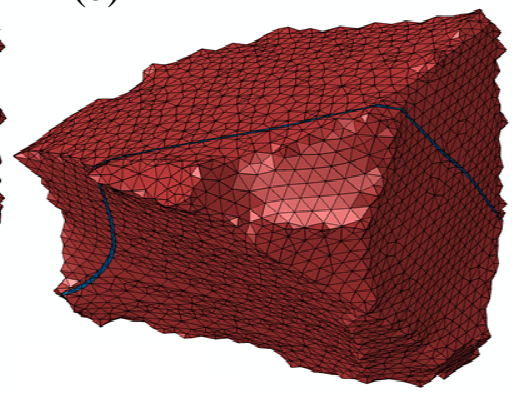

(c)

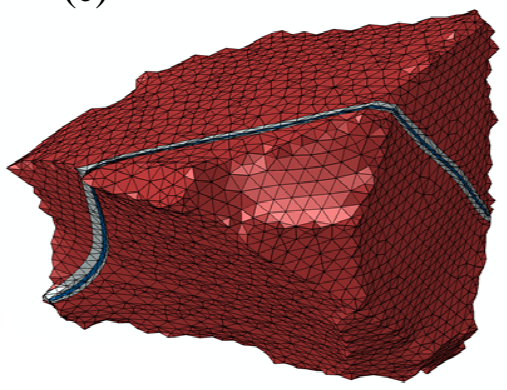

Figure 6 (a) FE mesh of the grain favorably oriented for twinning. (b) Parent and twin grain after creating the twin lamella of $1 \%$ volume fraction. (c) Parent grain and twin after twin growth from $1 \%$ to $3 \%$ volume fraction. Note the small change in shape of the grain under deformation. The original FE mesh of the parent grain has not changed substantially after the twin lamella is introduced except in close proximity to the twin lamella.

(a)

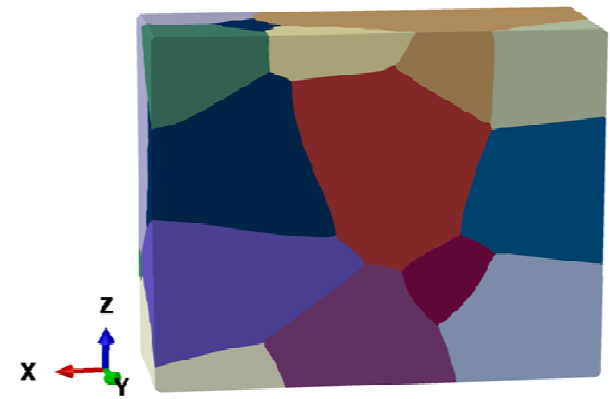

(b)

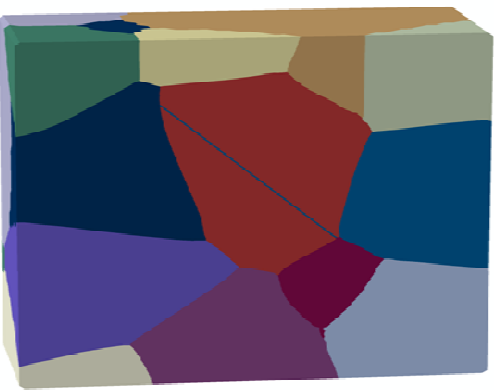

(c)

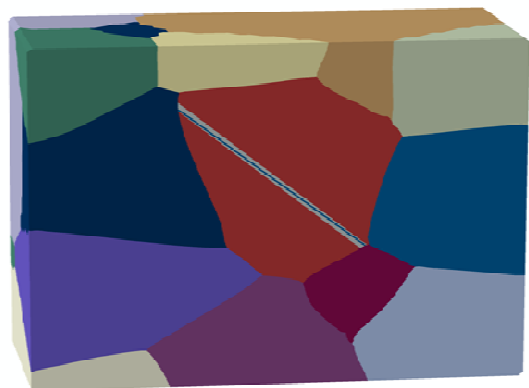

Figure 7 Twin formation and thickening with respect to the surrounding neighboring grains in the 3D model: (a) initial grain structure, (b) grain structure with $1 \%$ formed twin and (c) grain structure with $3 \%$ twin. 


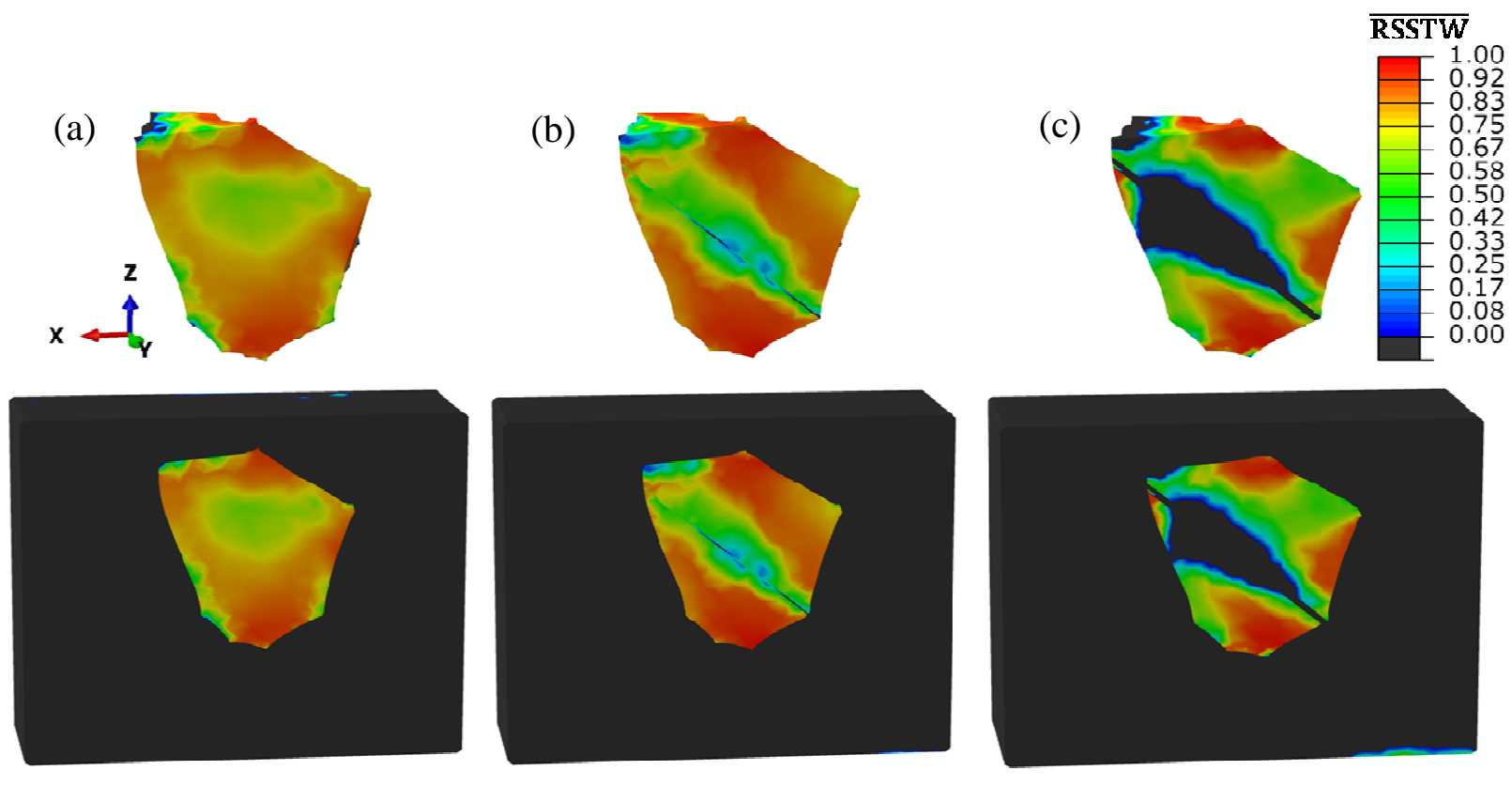

Figure 8 Distributions of normalized resolved shear stresses $(\overline{\text { RSSTW }})$ along the most dominant twin plane in the twin direction for different twin volume percentage (a) no twin, (b) $1 \%$ twin, and (c) $3 \%$ twin. Note that neighboring grains do not exhibit the propensity for twinning. 


\section{After continuous} deformation
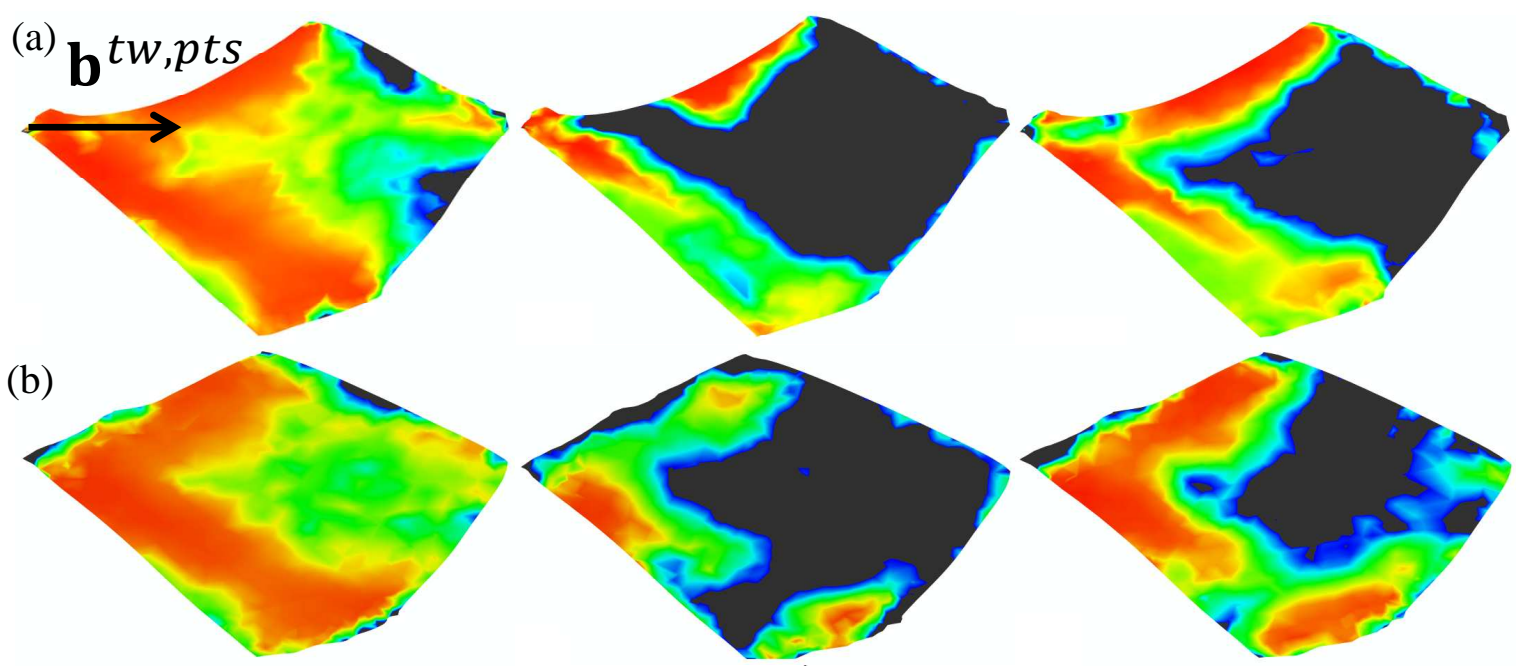

(c)
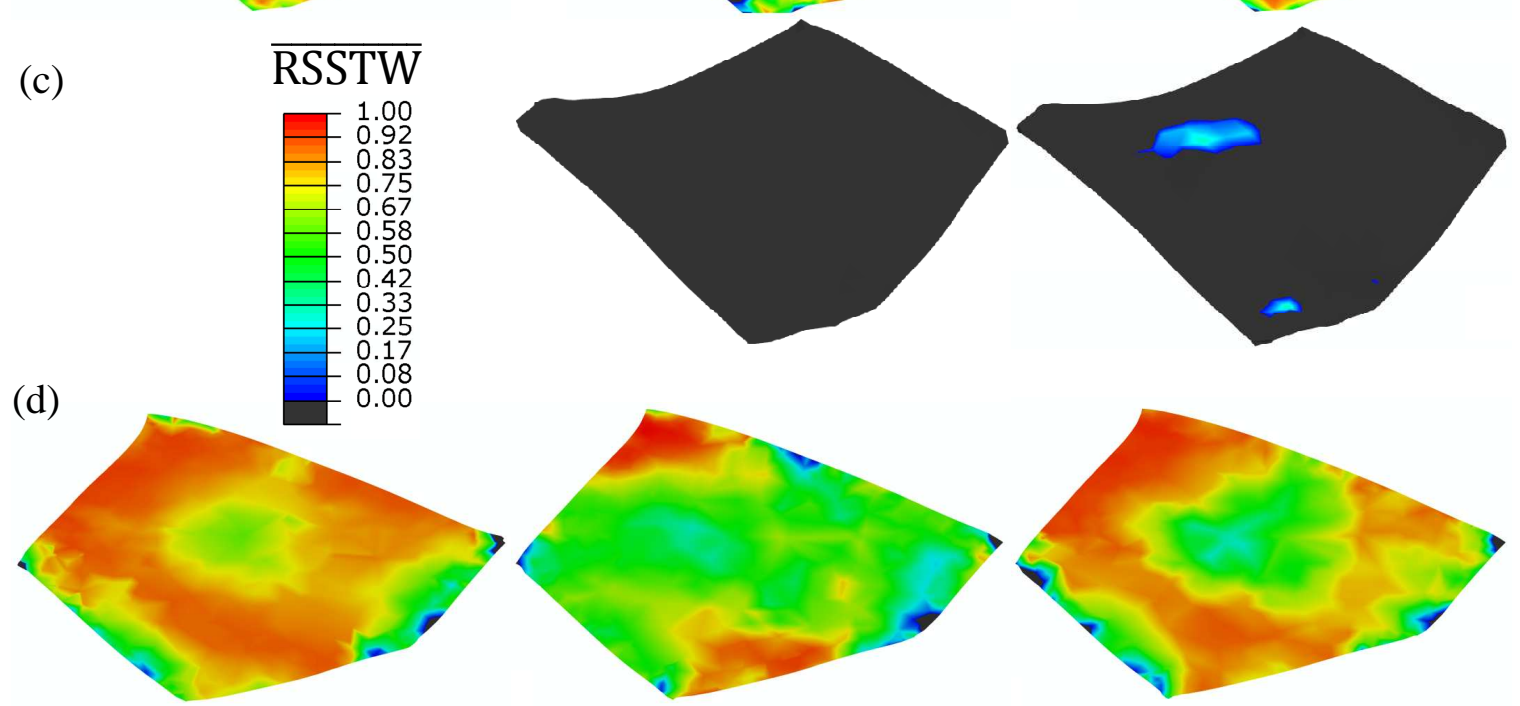

Figure 9 Distributions of normalized resolved shear stress $(\overline{\text { RSSTW }})$ along (a) the left interfacial planes between matrix and the twin lamella, (b) the right interfacial planes between matrix and the twin lamella, (c) a plane in the middle of the twin, and (d) slightly away in the matrix region from the right interface where the second twin lamella has formed. Different stages of deformation are shown as indicated in the figure. 
(a)

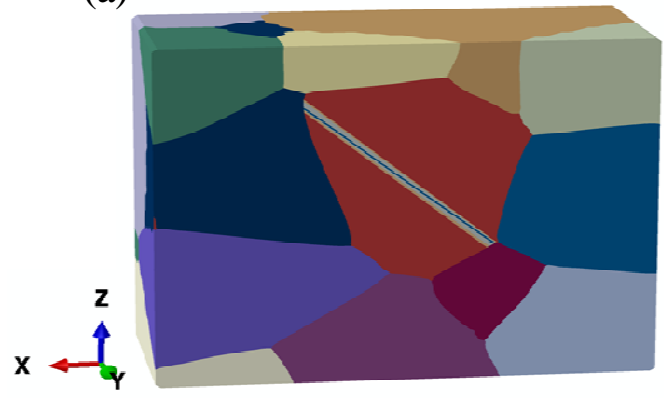

(b)

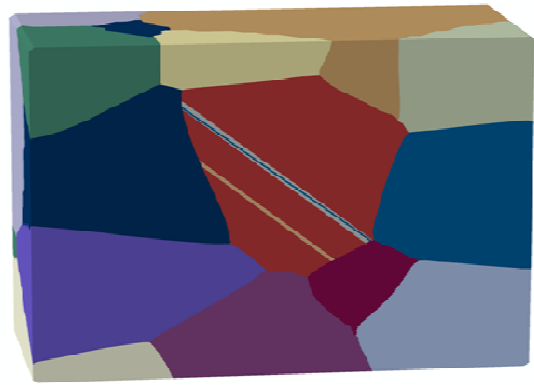

Figure 10 Grain structures with a parent grain containing (a) one 5\% twin lamella and (b) two lamellae one of $2 \%$ and another of $3 \%$ volume fraction.
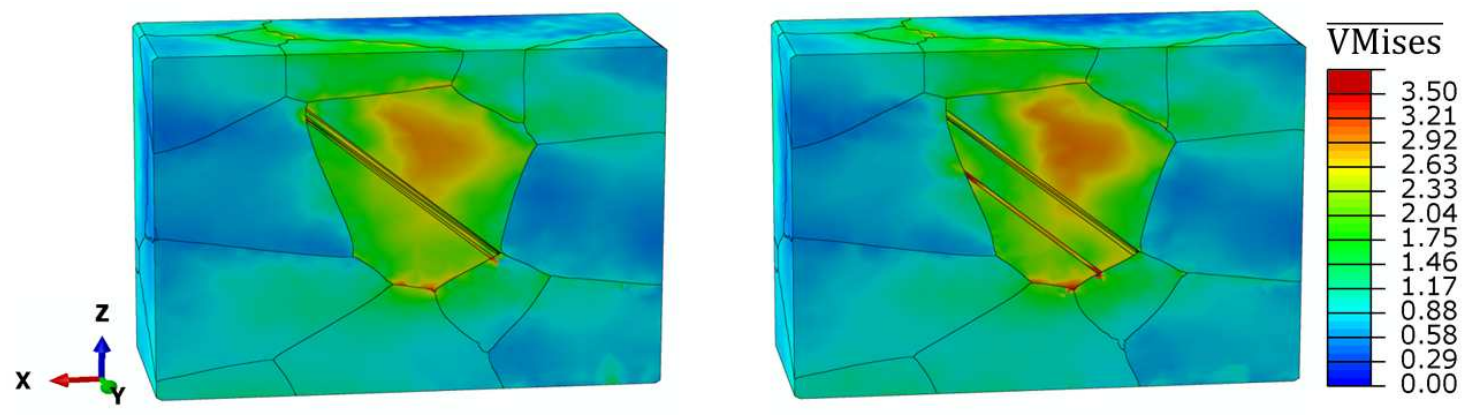

Figure 11 Distributions of normalized value of equivalent stresses (VMises) for 5\% twin volume fraction for one lamella twin and two twin lamellae.

(a)
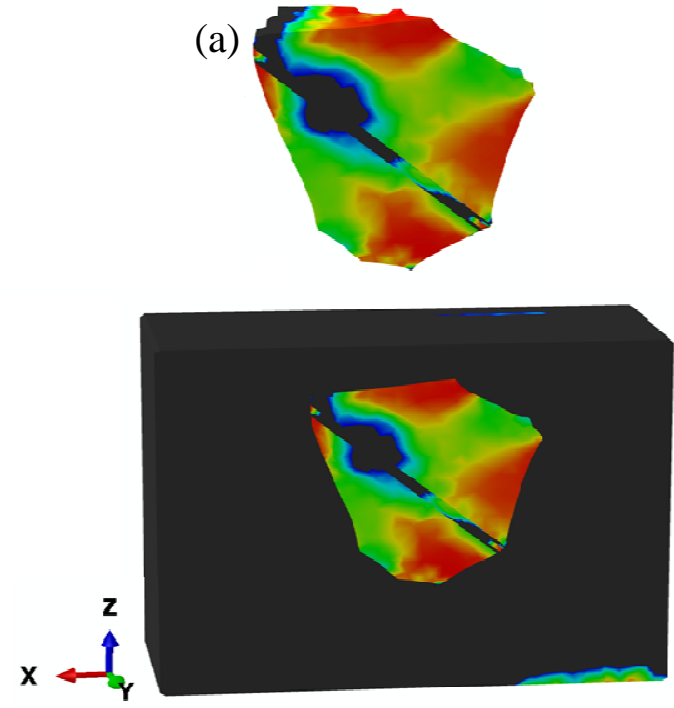

(b)
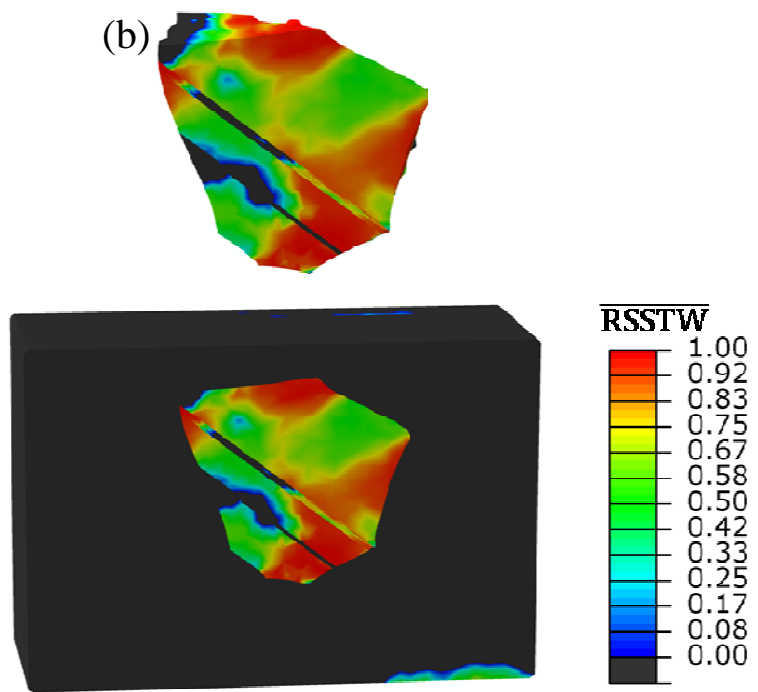

Figure 12 Distributions of normalized resolved shear stresses $(\overline{\text { RSSTW }})$ along the most dominant twin plane in the twin direction for different 5\% twin volume fraction (a) one lamella and (b) two lamellae. 
(a)

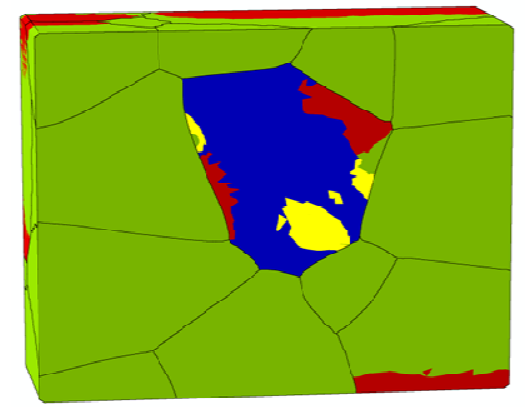

(b)

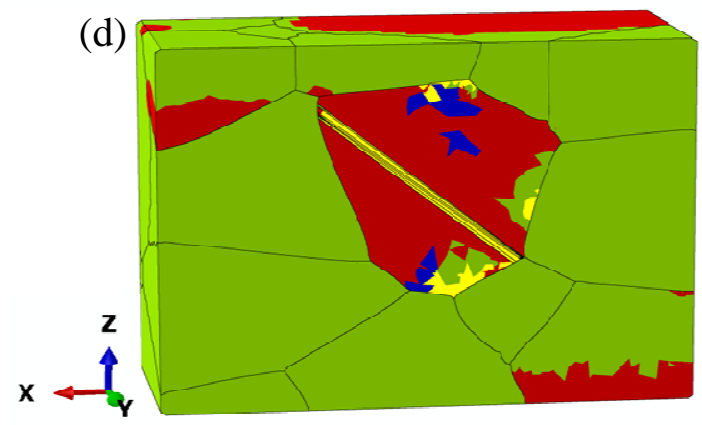

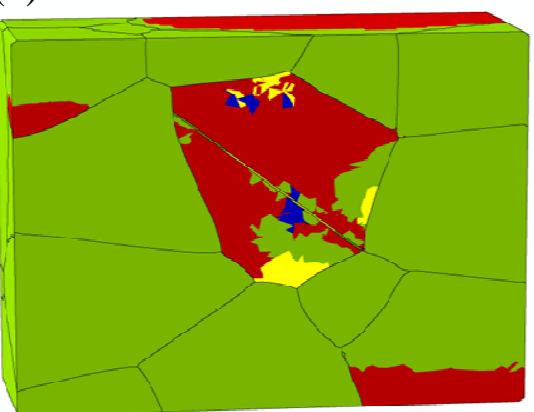

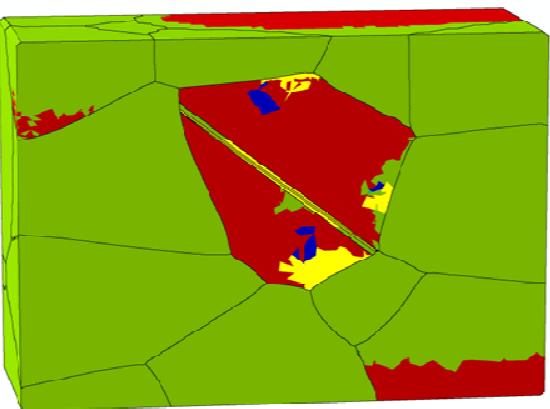

(e)

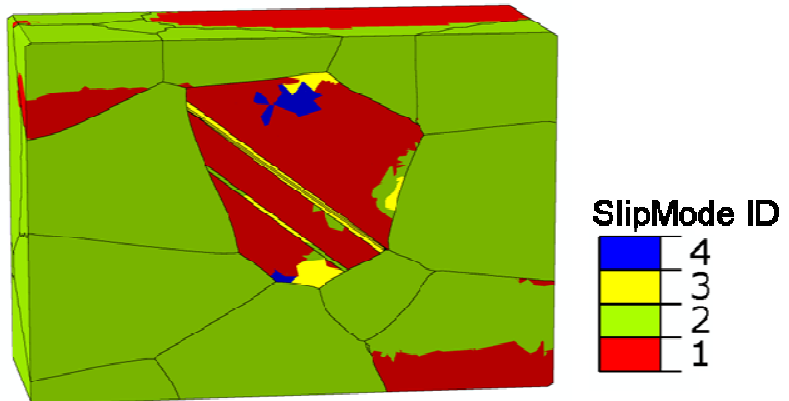

Figure 13 Distributions of the most active slip modes at (a) no twin, (b) $1 \%$ twin, (c) $3 \%$ twin, (d) 5\% one lamella twin and (e) 5\% two lamellae twin. Slip mode IDs: 1. (010)[100], 2. $\frac{1}{2}\{110\}<1 \overline{1} 0>, 3$. (001)[100], 4. $\left.\frac{1}{2}\{021\}<1 \overline{1} 2\right\rangle$. 
Table I Comparison of plastic work calculated over the volume indicated in the table with one lamella vs. two twin lamellae.

\begin{tabular}{|c|c|c|c|}
\hline $\begin{array}{c}\text { Work integral } \\
\text { (Eqns. 23 and 24) }\end{array}$ & $\Delta W_{1}\left[\frac{\mathrm{MJ}}{\mathrm{m}^{3}}\right] \times 10^{4}$ & $\Delta W_{2}\left[\frac{\mathrm{MJ}}{\mathrm{m}^{3}}\right] \times 10^{4}$ & $\Delta W\left[\frac{\mathrm{MJ}}{\mathrm{m}^{3}}\right] \times 10^{4}$ \\
\hline Grain cluster & 5.15 & 2.85 & 2.3 \\
\hline Parent grain + twin & 2.30 & 1.24 & 1.06 \\
\hline Parent grain & 1.49 & 0.74 & 0.75 \\
\hline Twin lamella & 0.81 & 0.5 & 0.31 \\
\hline
\end{tabular}

